\title{
Revealing cell populations catching the early stages of the human embryo development in naïve pluripotent stem cells
}

Marta Moya-Jódar"\#, Asier Ullate-Agote ${ }^{1,2 \#}$, Paula Barlabé ${ }^{1}$, Juan Roberto RodríguezMadoz $^{3,6}$, Gloria Abizanda ${ }^{1}$, Carolina Barreda ${ }^{1}$, Xonia Carvajal-Vergara ${ }^{1}$, Amaia VilasZornoza $^{2,6}$, Juan Pablo Romero ${ }^{2,4}$, Leire Garate ${ }^{3,6}$, Xabier Agirre ${ }^{3,6}$, Giulia Coppiello ${ }^{1}$, Felipe Prósper*1,3,5,6, Xabier L. Aranguren ${ }^{1 *}$.

${ }^{1}$ Program of Regenerative Medicine, Center for Applied Medical Research (CIMA), University of Navarra, Pamplona, 31008, Spain; Instituto de Investigación Sanitaria de Navarra (IdiSNA), Pamplona, 31008, Spain.

${ }^{2}$ Advanced Genomics Laboratory, Program of Hemato-Oncology, Center for Applied Medical Research (CIMA), University of Navarra, Pamplona, Spain.

${ }^{3}$ Hemato-Oncology Program, Center for Applied Medical Research (CIMA), IDISNA, University of Navarra, Pamplona, Spain.

${ }^{4} 10 x$ Genomics, 6230 Stoneridge Mall Road, Pleasanton, CA 94588, USA

${ }^{5}$ Hematology Department, Clínica Universidad de Navarra, University of Navarra, Pamplona, Spain

${ }^{6}$ Centro de Investigación Biomédica en Red de Cáncer (CIBERONC), Pamplona, Spain

\# co-first authors

* Correspondence: fprosper@unav.es; xlaranguren@unav.es

Keywords: Single-cell RNA-seq, Naïve state, Trophectoderm, 8CLCs

\section{SUMMARY}

Little is known about the heterogeneity existent in human naïve pluripotent stem cell (hPSC) cultures. In this study, we applied single-cell RNA sequencing to define heterogeneous cell populations present in human naïve 5iLAF cultures. While Epiblastlike cells represented the main cell population, noteworthily we detected a population with a trophectoderm-like gene expression profile, which could be subclustered according to three different stages of early trophectoderm maturation. Furthermore, we provided evidence for the existence of an 8-cell-like cell population, equivalent to the 8-cell human embryo. Thus, the 5iLAF naïve culture condition emerges as an excellent system to model gene regulation in the earliest stages of human embryogenesis, including zygotic genome activation, circumventing the inaccessibility and ethical issues inherent in the use of human embryos. 


\section{INTRODUCTION}

Embryonic development starts from a single totipotent cell called zygote, derived from the fertilized egg. Maternally derived RNA is used in the first phases of embryonic development, in particular, until the 2-Cell (2C) stage in mouse and the 8-Cell (8C) stage in human, when zygotic genome activation (ZGA) occurs, and the first wave of embryo genome transcription starts. In human, the first lineage differentiation events take place in the compact morula at day 4 , with the outer cells initiating a trophectoderm- (TE) specific transcriptional program. As a result, 5 days after fertilization, the blastocyst is segregated into the inner cell mass (ICM), which will give rise to the embryo proper, and the TE, which supports uterine implantation. By day 6, the blastocyst's ICM differentiates into the primitive endoderm ( $\mathrm{PrE})$, which will be a major constituent of the yolk sac, and the epiblast, which will form the fetus, while the TE will generate the extraembryonic tissues, including the placenta. Therefore, at the late blastocyst stage, prior to the implantation, these three main cell lineages are already determined. (Shahbazi, 2020).

Human pluripotent stem cells (hPSCs) can be obtained and maintained either in primed state, representing the in vitro counterpart of the post-implantation epiblast, or in naïve state, corresponding to the pre-implantation epiblast. Diverse protocols have been established to obtain hPSCs with naïve characteristics (Chan et al., 2013; Gafni et al., 2013; Guo et al., 2017, 2016; Liu et al., 2017; Takashima et al., 2014; Theunissen et al., 2014; Ware et al., 2014). Comparative transcriptional and molecular analyses have demonstrated that, among these protocols, the 5iLA, t2i/L+Gö and PXGL culture conditions produce cells with the highest resemblance to the in vivo pre-implantation epiblast (Takashima et al., 2014; Theunissen et al., 2014; Pastor et al., 2016; Stirparo et al., 2018; Theunissen et al., 2016).

Certain studies have identified different subpopulations of naïve hPSCs in 5iLAF cultures based on the expression levels of SSEA4 (Pastor et al., 2016); moreover, expression of the primitive endoderm (PrE) master gene GATA6 has been detected in different naïve cultures (Guo et al., 2016; Linneberg-Agerholm et al., 2019) and trophectoderm markers such as GATA3 and CDX2 have been identified in 5iLA cultures (Dong et al., 2020), suggesting the presence of different cell populations representing distinct cell lineages of the human pre-implantation embryo. However, a single cell transcriptomics study did not show significant heterogeneity in human embryonic stem cells (hESCs) in $\mathrm{t} 2 \mathrm{i} / \mathrm{L}+\mathrm{Gö} \mathrm{naïve}$ conditions (Messmer et al., 2019). Nevertheless, they interestingly identified a very small intermediate population presenting a gene expression profile that separate from both naïve and primed states (Messmer et al., 2019). On the other hand, there are significant evidences proving the existence of heterogeneous cell populations in mouse naïve PSCs cultures: cells equivalent to ICM (identified by the double expression of PDGFR $\alpha$ and CD31), PrE-precursors (PDGFR $\alpha^{+}$; CD31') (lo Nigro et al., 2017) and 2-cell-like cells (2CLCs) (Macfarlan et al., 2012) have been described. 2CLCs are the in vitro equivalent to the $2 \mathrm{C}$ stage mouse embryo and are characterized by the expression of genes from the Zscan4 family and MERVL retrotransposons, while showing downregulation of proteins associated with pluripotency, like Pou5f1, Sox 2 and Nanog. In addition, a cell population transitioning from naïve to 2CLCs state was identified, being characterized by Zscan4 genes expression, and lack of retrotransposon activation (Rodriguez-Terrones et al., 
2018). Therefore, to fill the void of knowledge regarding hPSCs heterogeneity, in the present study we analyzed by single-cell RNA sequencing naïve human induced PSCs (hiPSCs) under 5iLAF culture conditions, finding different cell populations which capture distinct stages of human embryo development, from the $8 \mathrm{C}$ stage to the periimplantation stage.

\section{RESULTS}

\section{Identification of cell heterogeneity in naïve hiPSC cultures}

To determine the cell heterogeneity of our 5iLAF naïve culture we performed single-cell RNA sequencing (scRNA-seq) and unsupervised clustering analysis of 3,652 cells, obtaining seven clusters (Figure 1A). First, we validated the naïve identity of our cells by integrating our data with previously published results (Liu et al., 2020), where the process of cell reprogramming from fibroblasts to iPSCs in primed and in naïve (RSeT and $\mathrm{t} 2 \mathrm{i} / \mathrm{L}+\mathrm{Gö}$ ) conditions was analyzed at single-cell resolution. Our sample clustered next to the naïve cells of this study and presented high score for naïve and epiblast gene signatures (Figure S1). These results were confirmed by morphology (Figure S2A), protein expression (Figure S2B-D), and a profound genome hypomethylation in LINE1 CpG sites (Figure S2E). Consistently, primed markers were not expressed in any of the seven clusters identified in our culture, demonstrating the lack of residual primed cells, except for CD24, which was enriched in Cluster 5 (Figure 1B). Naïve and pluripotency markers associated with the human embryo epiblast, were homogeneously expressed in Clusters 0 to 3, with the exception of PRDM14, that was lower in Cluster 2 (Figure 1CD). On the other hand, some of these markers were downregulated in Clusters 4, 5 and 6 (Figure 1C-D). Additionally, correlation analysis of differentially expressed genes (DEG), showed high similarity between Clusters 0 to 3, while Clusters 4, 5 and 6 defined distinct cell populations (Figure 1E). Therefore, we considered Clusters 0 to 3, representing $80.5 \%$ of the total cells, as a single population which we named Epi-Cluster $0-3$. A comparison of the DEG from this cluster (Table S1) with publicly available stagespecific gene expression modules of human embryos from zygote to late blastocyst stage (Stirparo et al., 2018), based on data from three different single-cell datasets (Blakeley et al., 2015; Petropoulos et al., 2016; Yan et al., 2013) showed that the majority of DEG $(53.5 \%)$ were specific to either late $(39.3 \%)$, or early $(14.2 \%)$ blastocyst's ICM (Figure $1 F)$. In addition, we analyzed the expression of transposable elements (Table S2), as their transcription is regulated in a stage-specific manner during human early embryogenesis (Göke et al., 2015). In our Epi-Cluster 0-3 we observed high expression of SVA family members, as well as LTR5-Hs and HERVK-int elements (Figure 1G), which are characteristic of morula and early blastocyst stage, in line with previously described naïve cells-specific transposable elements (Theunissen et al., 2016).

\section{Presence of trophectoderm-like cells in naïve hiPSC cultures}

We found that cells in Cluster 5, which represent the $4.4 \%$ of total cells, displayed the highest scores for a previously described TE signature (Petropoulos et al., 2016) (Figure 2A). In agreement, known TE-associated genes (Petropoulos et al., 2016; Xiang et al., 
2020) were enriched in this cluster (Figure 2B). Henceforth, we will refer to Cluster 5 as TE-like Cluster. RNA velocity analysis indicated that TE-like cells arose from naïve cells of Epi-Cluster 0-3 (Figure 2C). This finding is consistent with the described capacity of human naïve cells to differentiate into the TE lineage under defined culture conditions (Cinkornpumin et al., 2020; Dong et al., 2020; Guo et al., 2021; Io et al., 2021; Liu et al., 2020). The presence of TE-like cells in our naïve culture was confirmed by the detection of $\mathrm{GATA}^{+}$cells by immunofluorescence staining (Figure 2D). Interestingly, within the TE-like Cluster, we were able to observe a few scattered cells expressing primitive endoderm (PrE)-specific genes (Stirparo et al., 2018): PDGFRA, COL4A1, GATA6, GATA4 and SOX17 (data not shown). The possible existence of PrE-like cells in naïve 5iLAF cultures is in line with the previously reported expression of GATA6 in human t2iLGÖY naïve cultures (Guo et al., 2017, 2016; Linneberg-Agerholm et al., 2019). Furthermore, PrE-like cells have been described in mouse ES cultures (lo Nigro et al., 2017).

\section{hiPSCs naïve culture as a model of early TE specification process}

Analysis of defined TE-associated markers showed heterogeneous expression within cells of the TE-like Cluster (Figures 2A and 3A). Accordingly, an unsupervised subclustering analysis revealed three subpopulations (Figure 3B). To determine possible differences in TE maturation stage between them, we scored the cells against the GATA2 and NR2F2 genes modules, recently described for early developmental stages of the human embryo (Meistermann et al., 2021). According to this study early TE cells (B3 stage) are GATA2module $^{+}$and NR2F2-module ${ }^{-}$, while mature TE cells (B4-B6 stage) are GATA2-module ${ }^{+}$ and NR2F2-module ${ }^{+}$(Meistermann et al., 2021). When applying the GATA2 module to our 5iLAF sample, we found the highest scores throughout the TE-like Cluster (Figure 3C). However, cells of Cluster 5_0 had relatively lower scores for this module, and mainly had negative scores for the NR2F2 module (Figure 3C), a first proof of their early maturation stage. Additionally, this cluster expressed epiblast markers such as DPPA3, $D P P A 5, D N M T 3 L$ and $A L P G$ at higher level compared to the other two subclusters (Table S1). Co-expression of epiblast and early TE genes is a hallmark of TE transition in human embryonic development (Niakan and Eggan, 2013; Stirparo et al., 2018), and thus we termed Cluster 5_0 as Transition-TE-like Cluster. Cluster 5_1 and Cluster 5_2 could be distinguished by differences in scores for the NR2F2-module, being higher in the latter cluster (Figure 3C). Therefore, we named Cluster 5_1 as Early-TE-like Cluster and Cluster 5_2 as Mature-TE-like Cluster. To corroborate these results, we applied signatures derived in vitro for the different steps of the differentiation process of TE cells from pre-cytotrophoblasts stage (pre-CTBs, corresponding to developmental day 6-7 of the human embryo; D6-7) to extravillous trophoblasts (EVTs; D14) (Xiang et al., 2020). Cells with the highest scores for the pre-CTBs (D6-7) signature were found in the Transition- and Early-TE-like Clusters, while cells in the Mature-TE-like Cluster presented the highest scores for the Early syncytiotrophoblast (STB; D8-10) signature (Figure 3D). On the other hand, cells from later stages of placental differentiation were not present, according to the low scores obtained for their corresponding signature (data not shown) and the absence of placental polypeptide hormones expression (Figure S3A). 
Therefore, these results confirm that the three subclusters found in the TE-like Cluster define TE-like cells at different maturation stages and thus naïve hiPSCs in 5iLAF medium can be a model to study the transition from epiblast cells to early and mature TE cells. For instance, the modulation of the transcription factors (TFs) differentially expressed in our TE-like subclusters (Figure S3B-C and Table S1) could elucidate their role in the TE-differentiation process. Moreover, in our 5iLAF cultures we retrieved 38 transposable elements differentially expressed in the TE-like Cluster, when compared to the rest of cells. Between them, we identified repetitive sequences from the LINE1, Alu and LTR families (Figure 3E-F and Table S2). Noteworthily 86 transposable elements were differentially expressed in Early- or Mature-TE-like subclusters compared to the other clusters, suggesting a specific role of those sequences in the maturation process of TE cells. Between the previously described TE-specific transposable elements for example, we found that LTR30, LTR10A (Liu et al., 2019) and LTR2B, were enriched in the Early-TE-like Cluster. On the other hand, LTR3A (Liu et al., 2019) (Liu et al., 2019) HERVK3-int and LTR10B2 were enriched in the Mature-TE-like Cluster (Table S2). Finally, transposable elements known to be involved in placental development (Senft and Macfarlan, 2021) such as endogenous retroviruses ERVW-1 and ERVFRD-1 were enriched in the Mature TE-like Cluster (Table S1). Thus, the study of transposable elements could help to elucidate the process of TE-maturation.

Maturation of TE cells is associated with specific metabolic requirements (Kaneko, 2016; Posfai et al., 2019), so next we analyzed the metabolic transcriptome in the different subclusters. Interestingly, pathways related to energy production were enriched in EarlyTE-like Cluster (Figure S3D and Table S1), in agreement with the high ATP consumption of these cells in vivo, required to expand the blastocoel cavity (Houghton et al., 2003). Meanwhile, Mature-TE-like Cluster was enriched in pathways related to steroid hormone synthesis/metabolism and regulation of angiogenesis, all functions needed to allow proper implantation of the embryo into the uterine wall (Figure S3D and Table S1). Finally, molecules playing a key role in the embryo attachment to the endometrium (Idelevich and Vilella, 2020) were observed in Mature-TE-like cells, such as PGF, CGA, TGFB1, VEGFA (Table S1).

In conclusion, TE-like cells present in naïve cultures mimic the early stages of TE development and could be an ideal model to study the transition from epiblast to TE, TE maturation, and the role of these cells in the implantation process in vitro.

\section{Identification of 8-cell like cells (8CLCs) in 5iLAF culture}

Interestingly, in Cluster 6 of our analysis, which corresponds to $1.7 \%$ of the total cells, we found cells expressing ZSCAN4 (Figure 4A), a defining gene of the 8C stage human embryo (Stirparo et al., 2018). Additionally, in this cluster we detected expression of DUXA, PRAMEF1-2, LEUTX, KLF17, and other markers associated with the human 8C stage (Jiang et al., 2002; Maeso et al., 2016; Stirparo et al., 2018; Töhönen et al., 2015; Wang et al., 2018) (Figure 4A and Table S1). On the other hand, we observed significantly lower expression levels of NANOG, SOX2, and PRDM14 compared to the Epi-Cluster 0-3, but not ZFP42 or POU5F1 (Figure 1C-D). This is in accordance with previous studies describing the downregulation of pluripotency-associated markers such 
as Nanog, Sox2, Prdm14 and Tfap2c, but not Pou5f1 during the transition from ESCs to 2CLCs in mouse cultures (Rodriguez-Terrones et al., 2018). The expression of ZFP42 together with ZSCAN4 supports the notion that Cluster 6 does not emerge from a process related to cell differentiation, since this process requires loss of ZFP42 expression (Rodriguez-Terrones et al., 2018). To further determine to which developmental stage of the human embryo Cluster 6 corresponds to, we compared its DEG to human embryo transcriptomic data (from zygote to late blastocyst stage; Stirparo et al., 2018). From the annotated genes (Stirparo et al., 2018), we observed that $40 \%$ were associated with the $8 \mathrm{C}$ stage and $\sim 20 \%$ with compact morula (Figure 4B). Additionally, applying the DUXA module (Meistermann et al., 2021) to the 5iLAF sample, we found that Cluster 6 was the one with the highest score (Figure 4C). The DUXA module comprises genes associated with the 8C and morula stage in humans (Meistermann et al., 2021). Accordingly, when applying the DUX4-induced signature (Hendrickson et al., 2017) to our data, we observed that Cluster 6 was enriched for these genes (Figure 4D). DUX4 overexpression in hiPSC activates cleavage-specific genes (Hendrickson et al., 2017). These data show that Cluster 6 has a gene expression profile similar to the $8 \mathrm{C}$ human embryo, therefore we termed it $8 \mathrm{C}$-like Cluster.

As the expression of MERVL retrotransposons is a hallmark of 2 CLCs in mouse (Macfarlan et al., 2012), we also analyzed transposable elements expression in the 8Clike Cluster. In this cluster we observed a significantly higher proportion of reads corresponding to retroposons, LTRs, SINEs and LINEs families compared to Epi-Cluster 0-3 (Figure 4E). Additionally, transposable elements known to be expressed between 8C and morula stages in human embryos such as the SVA retroposons, LTR5-Hs, and LTR7Y (Göke et al., 2015; Liu et al., 2019) were enriched in the 8C-like Cluster, as well as 8C-specific transposable elements such as HERVK-int, LTR12C and MLT2A1 (Göke et al., 2015; Grow et al., 2015; Liu et al., 2019). Moreover, we found that Mer68-int and LTR1A2, which have not been previously described as being expressed in early phases of embryonic development, were highly expressed in the 8C-like Cluster.

Noteworthily, MLT2A1, the most specific repetitive sequence to the $8 \mathrm{C}$ human embryo in vivo (Göke et al., 2015), was among the most enriched in the 8C-like Cluster (Fig 4F$\mathrm{H}$ and Table S2). Importantly, ZSCAN4 expression correlated with MLT2A1 expression (Figure 4I). Thus, we defined the $Z S C A N 4^{+} / \mathrm{MLT} 2 \mathrm{~A} 1^{+}$cells found in the $8 \mathrm{C}$-like Cluster as 8CLCs. In mPSC cultures, Zscan4 $4^{+} \mathrm{MERVL}^{+} 2 \mathrm{CLCs}$ range from 0.1 to $0.5 \%$ of the total cells (Macfarlan et al., 2012). Accordingly, in our naïve 5iLAF sample, we observed a similar proportion of 8CLCs $(\sim 0.3 \%)$. Finally, we confirmed by immunofluorescence staining the existence of $\mathrm{ZSCAN}^{+}$cells in 5iLAF cultures, while they were absent in primed cultures (Figure 4J).

In conclusion, these data prove the existence of 8CLCs in 5iLAF cultures. These cells could be an ideal in vitro platform to study the earliest stages of the human embryo development, including the ZGA events.

\section{Presence of a cell population characterized by cell cycle arrest}

Cluster 4 , representing $13.4 \%$ of the total cells, expressed several epiblast markers, although could be distinguished by a very low expression of NANOG, SOX2, PRDM14 
and KLF4 (Figure 1C-D). The most prominent characteristic found in this cluster was the high percentage of cells in cell cycle arrest (G1 phase, Figure 5A). In fact, the most significantly enriched gene sets for this cluster are related to response to DNA damage and cell cycle arrest (Figure 5B). When studying the differential expression of transposable elements in Cluster 4, we found LINE elements of the L1-subfamily, among others (Figure 5C-D). Interestingly, L1 elements play an important role in genomic instability and cancer and L1 retrotransposition induces cell stress via DNA response to damage (Grundy et al., 2021). The transcriptional profile of Cluster 4 suggests cell damage and is consistent with the known detrimental effects on genetic and epigenetic stability induced by naïve conditions on cultured hPSCs (Liu et al., 2017; Pastor et al., 2016; Theunissen et al., 2014).

\section{Heterogeneous contribution of hiPSCs to human-mouse chimera}

Epiblast-like naïve PSCs microinjected in a host embryo are known to contribute to the ICM (Beddington and Robertson, 1989). To functionally prove the existence of cells corresponding to distinct developmental stages in our cultures, we conducted chimera formation experiments. We microinjected 8-10 5iLAF cells, labelled with tdTomato, into morula-stage mouse embryos and cultured them for $48 \mathrm{~h}$ in vitro (Figure 6A-B). Quantification at $24 \mathrm{~h}$ showed that part of the microinjected cells did not engraft in the host embryo, as we observed a decrease in cell number at this time point, whereas we detected cell proliferation at $48 \mathrm{~h}$, as indicated by an increased number of cells (Figure 6C). Strikingly, at this time point, in addition to finding human cells in the ICM, we also observed a fraction of cells contributing to the TE (Figure 6D-F). TE identity of the human cells located in the outer layer of the chimeric embryos was confirmed by immunofluorescence for the GATA3 marker (Fig. 6D-E). These results further support the existence of cell heterogeneity within human 5iLAF cultures.

\section{DISCUSSION}

With this work we described the heterogeneity present in human naïve 5iLAF cultures, revealing four main cell populations with distinct characteristics. While the expression profile of $80 \%$ of the cells, here named Epi-Cluster 0-3, defined them as the in vitro counterpart of the late blastocyst epiblast cells, which corresponds to the gold standard definition of naïve, we also revealed the presence of TE-like cells and 8CLCs, as well as a population of Epiblast-like cells in cell cycle arrest.

The TE-like Cluster is characterized by a gene expression profile comparable to the early TE of the human embryo (D6-9) and expresses at high levels TE-specific transposable elements. This population represents $4.4 \%$ of the cells. Such population of cells was not previously found in naïve PXGL conditions, possibly as a consequence of the presence of WNT and aPKC inhibitors, which block the access to TE differentiation in this medium (Guo et al., 2021). RNA velocity trajectory analysis showed that cells of the TE-like Cluster arise from naïve cells. This is in line with the known potential of naïve hPSCs to give rise to trophoblast stem cells (hTSCs) in a defined media (Okae et al., 2018), being hTSCs the in vitro counterpart of the trophoblast at D8-12 (Castel et al., 2020; Cinkornpumin et al., 2020; Dong et al., 2020; Guo et al., 2021; Io et al., 2021). 
Interestingly, TE-like cells found in our naïve cultures presented a heterogeneous gene and transposable elements expression profile, that allowed us to subdivide them into three subclusters, corresponding to three early extraembryonic tissue maturation stages: transitional, early TE and mature TE. Thus, while hTSCs can be used to study trophoblast lineages differentiation (Castel et al., 2020; Cinkornpumin et al., 2020; Dong et al., 2020; Guo et al., 2021; Io et al., 2021), TE-like cells in 5iLAF cultures, which resemble the trophectoderm of the blastocyst at day D6-9, may be a suitable model to shape the role of the different genetic determinants of TE formation and its early maturation.

TE cells contribute to the process of implantation by modulating endometrial receptivity (Posfai et al., 2019). In the TE-like Cluster, we observed factors previously described to take part in this process and we unraveled new candidates that may play an important role in trophoblast to endometrium communication as, for instance SEMA4C (Table S1), whose receptor, PLEXIN B2, has been reported to participate in endometrial integrity (Singh and Aplin, 2015). Since trophoblast dysfunction leads to complications during pregnancy, such as pre-eclampsia and intrauterine growth restriction, the study of TE-like cells present in patient-derived naïve iPSC cultures would facilitate the identification of the molecular players inducing these complications.

Human 5iLAF cells microinjected at the morula stage into mouse embryos showed contribution to both ICM and TE, with TE-contribution present in $36 \%$ of the chimeric embryos at $48 \mathrm{~h}$ post-injection. The identification of hiPSCs TE-contribution importantly confirms the existence of cells with in vivo TE-differentiation capacity in human naïve culture. We hypothesize that these cells are more likely to be derived from the TE-like Cluster, as the proportion of 8CLCs in our culture is too low to justify such a high frequency of TE-contribution. Accordingly, in chimera experiments with mouse PSCs, where a population of 2CLCs (Macfarlan et al., 2012) but not a TE-like population has been described, the contribution of microinjected cells to the TE was extremely rare (Beddington and Robertson, 1989). Nevertheless, to prove this hypothesis, it would be necessary to identify surface markers that allow the isolation of TE-like cells from the naïve bulk and to perform chimera formation experiments with these isolated cells.

Noteworthily, 8C-like Cluster defines a population of cells enriched with the expression of $8 \mathrm{C}$ embryo specific genes and transposable elements, while showing downregulation of pluripotency-associated genes like PRDM14,SOX2 and NANOG. In this population we detected the existence of $Z S C A N 4^{+} / \mathrm{MLT} 2 \mathrm{~A} 1^{+}$cells, which present a gene and transposable elements expression characteristics of the $8 \mathrm{C}$ stage of the human embryo in vivo. These 8 CLCs represent $\sim 0.3 \%$ of the total cells, consistent with the described proportion of 2CLCs in mouse cultures (Macfarlan et al., 2012). This discovery is in line with the recent description of an analogous 8CLCs population in naïve PXGL medium (Taubenschmid-Stowers et al., 2022) and opens the door to the use of naïve PSCs as a model to study key pathways determining the origin and developmental potential of 8CLCs. The in vivo counterpart of these cells is characterized by a higher state of pluripotency compared with ICM cells, therefore it would be of interest to test the chimeric capacity of 8CLCs with functional experiments. However, the isolation of this cell population and its expansion would be needed in order to perform these studies. For 
that, it will be of pivotal importance to identify suitable surface markers or generate reporter cell lines and to find key molecules to obtain an adapted culture media.

Unlike mouse PSCs, which are genetically stable after prolonged culture (Cervantes et al., 2002; Suda et al., 1987), human naïve PSCs are prone to acquire mutations, chromosomal abnormalities and epigenetic aberrations (Pastor et al., 2016; Theunissen et al., 2014) and therefore is still necessary to revise the naïve culture conditions to improve cell fitness. Accordingly, in our study we have observed that cells of Cluster 4 are in cell cycle arrest and express genes related to response to DNA damage. This observation reinforces the notion that renewed refinement of 5iLAF medium is required to improve cell stability. Stress response related genes enriched in Cluster 4, such as DDIT3 and CDKN1A (Ock et al., 2020) (Table S1), could be used as markers to monitor cell integrity upon modification of the culture conditions.

In summary, our study describes the heterogeneity present in 5iLAF naïve hiPSCs. Distinct populations corresponding to 8CLCs and TE have been found to coexist with epiblast-like (naïve) cells in these cultures. Hence, 5iLAF condition could be used as a model to investigate human embryo development from the ZGA event to the periimplantation period, as well as to broaden the understanding of stem cell biology, thus avoiding the use of human embryos and the associated ethical issues.

\section{ACKNOWLEDGMENTS}

This study was supported by the Instituto de Salud Carlos III co-financed by European Regional Development Fund-FEDER “A way to make Europe” Red de Terapia Celular TERCEL (RD16/0011/0005) and Centro de Investigación Biomédica en Red de Cáncer CIBERONC (CB16/12/00489), and co-financed by European Union NextGenerationEU. Redes de Investigación Cooperativa Orientada a Resultados en Salud RICORS (RD21/0017/0009 and RD21/0017/0019), the Ramón y Cajal State Program (MINECO/FSE, RYC-2015-17233), Ministerio de Ciencia e Innovación/Agencia Estatal de Investigación/FEDER, (UE/RTI2018-064485-B-I00), MINECO/FSE, (IJCI-201733070) and Gobierno de Navarra I+D 2020 Co-Funded by FEDER Funds (0011-13652020-000293 and 0011-1365-2020-000287).

\section{AUTHOR CONTRIBUTION}

M.M.J. Generated and characterized naive hiPSCs, performed single-cell experiment, performed mouse-human chimera experiments, analyzed data, and wrote the manuscript. A.U. Performed bioinformatic analysis of the data, and wrote the manuscript. P.B. Provided assistance with experiments and edited the manuscript. A.V., G.A., C.B, and L.G. provided assistance with experiments. J.P.R. provided assistance with bionformatic analysis. J.R.R., X.C.V and X.Ag. provided materials and technical advice and edited the manuscript. G.C. discussed the data and wrote the manuscript. F.P. and X.A. conceived the research, provided funding, supervised the experiments, analyzed the data, and wrote the manuscript.

\section{AUTHOR DISCLOSURE STATEMENT}

J.P.R is an employee and shareholder of 10x Genomics. 


\section{FIGURE LEGENDS}

Figure 1: Overall analysis of cell heterogeneity in human naïve 5iLAF culture.

(A) Uniform manifold approximation and projection (UMAP) of the 5iLAF-cultured hiPSCs (3,654 cells). Cells are color-coded according to unsupervised clustering analysis. (B-D) Violin plots showing single cell log-normalized gene expression of selected primed, naive and pluripotency markers in each cluster. Color code as in panel A. (E) Heatmap representing the correlation between the different clusters, considering differentially expressed genes in at least one cluster against the rest. (F) Proportion of differentially expressed genes in Epi-Cluster 0-3 corresponding to each stage-specific gene expression module of the human embryo at early developmental stages obtained from Stirparo et al., 2018 and listed in their Table S6. Genes differentially expressed in Epi-Cluster 0-3 that were not specific to any developmental stage or were not annotated were remove from the analysis. (G) UMAP representations of the normalized expression of naïve-specific transposable elements. A contour delineates Epi-Cluster 0-3.

\section{Figure 2: TE-like cells in human naïve 5iLAF culture}

(A) UMAP representation of scores from a TE signature including TE-specific markers that are common between Petropoulos et al., 2016 and Xiang et al., 2020 studies. A contour delineates Cluster 5. (B) Heatmap representing scaled gene expression of the 111 genes from the TE signature. To facilitate visualization of the data, 200 cells from each cluster were randomly selected. Cells are ordered by cluster membership. (C) UMAP plot showing RNA velocities as streamlines. (D) Immunofluorescence staining of GATA3 (in green) in human naïve (upper panels) and human primed (lower panels) cultures. Human cells are labelled with tdTomato (in red) and Hoechst was used as a nuclear counterstain. Scale bars, $50 \mu \mathrm{m}$.

Figure 3: Analysis of the three different stages of TE maturation present in the TElike Cluster. (A) UMAP representing log-normalized expression of a selected group of TE-associated markers. A contour delineates TE-like Cluster. (B) UMAP representation after subclustering the TE-like Cluster. Cells are color-coded according to unsupervised clustering analysis. The dashed box corresponds to the UMAP region shown in the upcoming panels $C$ and D. (C) UMAP representation of scores from the GATA2 gene module and the NR2F2 gene module signatures from Meistermann et al., 2021. A contour delineates each subpopulation from the TE-like Cluster. Violin plots of the scores grouped by TE-like subpopulations are depicted underneath, with a dashed line at score zero for the NR2F2 module. (D) UMAP representation of gene signature scores from different trophoblast lineages obtained from Xiang et al., 2020: pre-CTB (days 6-7), postCTBs (days 7-8) and early STBs (days 8-10). A contour delineates each subpopulation from the TE-like Cluster. Violin plots of the scores grouped by TE-like subpopulations are depicted underneath. (E) Dot plot representing scaled average expression of the top differentially expressed transposable elements in each of the TE-like subclusters. The size of the dot represents the proportion of cells expressing such element in each cluster. (F) UMAP representing the log-normalized expression in TE-like subclusters of selected transposable elements. A zoom-in of the TE-like cluster is displayed, with a contour 
delineating each subpopulation. Violin plots grouped by TE-like subpopulations are shown underneath the zoom-ins. The color code from the legend is the same for the violin plots in panels $\mathrm{C}, \mathrm{D}$ and $\mathrm{F}$.

\section{Figure 4: 8CLCs in human naïve 5iLAF culture.}

(A) Zoom-ins of the UMAP region including the 8C-like Cluster representing the lognormalized expression of selected $8 \mathrm{C}$ embryo associated markers. The zoom-ins correspond to the dashed region shown in the top left UMAP. A contour delineates the 8C-like Cluster. (B) Proportion of differentially expressed genes in the 8C-like Cluster corresponding to each stage-specific gene expression module of the human embryo at early developmental stages obtained from Stirparo et al., 2018 and listed in their Table S6. Genes differentially expressed in 8C-like Cluster that were not specific to any developmental stage or were not annotated were removed from the analysis. (C) UMAP representation of scores from the DUXA gene module from Meistermann et al., 2021. A contour delineates the 8C-like Cluster. (D) Heatmap representing scaled gene expression of 144 markers upregulated after DUX4 induction in hiPSCs from Hendrickson et al., 2017. All cells from 8C-like Cluster were represented, while to facilitate visualization of the data, 50 cells from each other cluster were randomly selected. Cells are ordered by cluster membership. (E) Boxplots showing the percentage of total UMIs corresponding to different transposable element classes (retroposons, LTRs, SINEs and LINEs) in cells from Epi-Cluster 0-3 and the 8C-like Cluster. (F) Dot plot representing scaled average expression of the top 10 differentially expressed transposable elements in the $8 \mathrm{C}$-like Cluster. The size of the dot represents the proportion of cells expressing such element in each cluster. (G) UMAP representation of log-normalized expression of transposable elements that are enriched in the $8 \mathrm{C}$-like Cluster. A contour delineates the $8 \mathrm{C}$-like Cluster. (H) Boxplots showing the percentage of total UMIs corresponding to the MLT2A1 LTR element in cells from Epi-Cluster 0-3 and 8C-like Cluster. Two-side Wilcoxon Rank Sum tests were performed to obtain statistical significance in the comparisons between clusters from panels E and $\mathrm{H}$ (I) Correlation analysis between ZSCAN4 and MLT2A1 expression in $\mathrm{ZSCAN4}^{+}$cells from the 8C-like Cluster. (J) Confocal microscopy images of immunofluorescence staining for ZSCAN4 (in green) in 5iLAF (on the left) and primed (on the right) hiPSCs. DNA was counterstained with Hoechst. Scale bars, $20 \mu \mathrm{m}$.

\section{Figure 5: Presence of naïve cells with arrested cell cycle in Cluster 4.}

(A) Proportion of cells in each cell cycle phase for the unsupervised clusters. (B) Selection of significant GO Biological Processes and WikiPathways terms from an overrepresentation analysis using DEG in Cluster 4 against the rest. (C) Dot plot representing scaled average expression of the top 10 differentially expressed transposable elements in Cluster 4. The size of the dot represents the proportion of cells expressing such element in each cluster. (D) UMAP representation of log-normalized expression of differentially expressed transposable elements in Cluster 4, delineated by a contour.

Figure 6. Contribution of human 5iLAF cells to the TE lineage in human-mouse chimera embryos. (A) Orthogonal projection of a mouse morula freshly microinjected 
with 10 human 5iLAF tdTomato ${ }^{+}$cells. (B) Engraftment of human cells within the mouse embryo after $48 \mathrm{~h}$ of in vitro culture, shown by orthogonal projection confocal image. (C) Graphical representation of the human cells present into mouse embryos over time. (D) Immunostaining of GATA3 (in green) in a mouse embryo microinjected with human 5 iLAF cells and cultured in vitro for $48 \mathrm{~h}$. (E) Orthogonal section from panel D. Boxed area is enlarged in the bottom right corner of the picture. The arrow indicates a tdTomato ${ }^{+}$ human cell expressing GATA3 in panels D and E. (F) Graphical representation of human cells contribution to ICM or TE $48 \mathrm{~h}$ post-microinjection. Dots represent microinjected embryos. Hoechst was used as a nuclear counterstain in A, B, D and E. Scale bars, $20 \mu \mathrm{m}$.

\section{STAR METHODS SECTION RESOURCE AVAILABILITY \\ Lead Contact}

Further information and requests for resources and reagents should be directed to and will be fulfilled by the Lead Contact, Xabier L. Aranguren (xlaranguren@unav.es).

\section{Animal models}

All animals were housed in specific pathogen free conditions with free access to food and water. All animal procedures were performed according to all state and institutional laws, guidelines and regulations. All studies were approved by the Ethics Committee for Animal Research at the University of Navarra and the Government of Navarra.

\section{Cell culture}

G15.AO human-induced pluripotent stem cell (hiPSC) line was used in this study (Zapata-Linares et al., 2016). Cells were routinely maintained on irradiated mouse embryonic fibroblast (iMEF) feeders in primed conditions: DMEM Knock-Out (Gibco \#10829-018), 20\% Knock-Out Serum Replacement (KSR, Gibco \#10828-028), 1\% GlutaMAX (Gibco \#35050-038), $1 \%$ non-essential amino acids (Gibco \#11140-035), 1\% Antibiotic-Antimycotic (Gibco \#15240-062), $0.1 \mathrm{mM} \beta$-mercaptoethanol (Gibco \#31350010) and $20 \mathrm{ng} / \mathrm{mL}$ human FGF-basic (Peprotech \#100-18B). After reaching 70\% confluence (5-7 days), cells were passaged by detaching them with $200 \mathrm{U} / \mathrm{mL}$ collagenase type IV (Gibco \#17104-019) for 10 minutes, followed by mechanical disruption of the colonies with cell scrapper and gentle pipetting. Cells were cultured in a humidified atmosphere at $37^{\circ} \mathrm{C}, 20 \% \mathrm{O}_{2}, 5 \% \mathrm{CO}_{2}$ in air.

5iLAF medium. HiPSCs were converted into naïve-like cells as described (Theunissen et al., 2014)with minor modifications of the media: briefly, 1:1 Neurobasal (Gibco \#21103-049), DMEM-F12 (Sigma-Aldrich \#D6421), 1X B27 Supplement (Gibco \#17504-044), 1X N2 Supplement (Gibco \#17502-048), 1\% GlutaMAX (Gibco \#35050038), $1 \%$ Non-Essential Amino Acids (Gibco \#11140-035), 1\% Antibiotic-Antimycotic (Gibco \#15240-062) 0.5\% Knock-Out Serum Replacement (KSR Gibco \#10828-028), 20 ng/mL recombinant human LIF (Peprotech \#300-05-100UG), 20 ng/mL recombinant human Activin A (Peprotech \#120-14-10UG), 8 ng/mL human FGF-basic (Peprotech 
\#100-18b-50UG), and the following small molecule inhibitors: $10 \mu \mathrm{M}$ Y27632 (Axon Medchem \#1683); $1 \mu \mathrm{M}$ WH-4-23 (Biovision \#2827-10), $1 \mu \mathrm{M}$ PD0325901 (Axon Medchem \#1408), $1 \mu$ M IM-12 (Sigma-Aldrich \#SML0084-5MG); $0.5 \mu$ M SB590885 (TOCRIS \#2650). Briefly, hiPSCs were passaged every 7-9 days by single-cell disaggregation with 1:1 TrypLE (Gibco \#12605-028) 1X PBS/0.25 mM EDTA (Invitrogen \#AM9260G) and plated on an iMEF feeder layer. Culture medium was supplemented with additional Y27632 inhibitor for the first $24 \mathrm{~h}$ at $5 \mu \mathrm{M}$. Cells were cultured in a humidified atmosphere at $37^{\circ} \mathrm{C}, 5 \% \mathrm{O}_{2}, 5 \% \mathrm{CO}_{2}$ in air and checked monthly for Mycoplasma contamination.

\section{Cells immunofluorescence staining}

Primed and naïve hiPSCs were cultured over iMEFs in 8-well chambers (Thermo Scientific Nunc\#177445) until they reached $60-70 \%$ of confluence. Then, they were fixed with $4 \%$ paraformaldehyde (PFA) solution in PBS for 30 minutes at $4{ }^{\circ} \mathrm{C}$, washed 3 times in PBS, permeabilized with PBS 0.1\% Triton ${ }^{\mathrm{TM}} \mathrm{X}-100$ (Fisher BioReagents \#BP151-500) for $1 \mathrm{~h}$ at $4^{\circ} \mathrm{C}$, washed again and then, blocked in PBS 1\% BSA (Sigma-Aldrich \# A3912$100 \mathrm{G}$ ) for 1 hour at $4^{\circ} \mathrm{C}$. Samples were stained with the following primary antibodies in blocking solution overnight at $4{ }^{\circ} \mathrm{C}$ : Anti-POU5F1 (1:50, Santa Cruz Biotechnology \#sc9081), Anti-SOX2 (1:50, Sigma-Aldrich \#AB5603), Anti-TFE3 (1:200, Merck - Sigma Aldrich \#HPA023881-100UL), Anti-hTFCP2L1 (1:200, R\&D systems \#AF5726), AntiGATA3 (1:100, Abcam \#ab199428), Anti-NANOG (1:50, ab80392, abcam), and AntiZSCAN4 (1:100, Origene \#TA800535). After rinsing three times with PBS to remove the unbound antibodies, samples were exposed to the corresponding Alexa Fluor ${ }^{\mathrm{TM}} 488$ goat anti-rabbit IgG secondary antibody (Invitrogen \#A11008), Alexa Fluor ${ }^{\circledR} 488$ donkey anti-goat IgG secondary antibody (Life Technologies \#A11055) or Alexa Fluor® 488 goat anti-mouse $\operatorname{IgG}$ secondary antibody (Invitrogen \#A11029), for 2 hours at $4^{\circ} \mathrm{C}$ in darkness (dilution 1:500 in blocking solution). After 3 washing steps with PBS, nuclear counterstain was performed with Hoechst 33342 trihydrochloride (Invitrogen \#H21492) at 1:800 dilution for 30 minutes at $4{ }^{\circ} \mathrm{C}$ in darkness. Images were recorded on a Confocal Scanning Laser Microscope (Zeiss LSM 800) and ZEN 2.3 system software. Composite images were obtained with ZEN 2.3 system software.

\section{Flow Cytometry and FACS sorting}

Pluripotency-, primed-, and naïve-associated markers were analyzed by FACS. Briefly, hiPSCs were cultured up to $70 \%$ confluence and washed once with PBS before singlecell dissociation. Then, hiPSCs were dissociated by incubation with TrypLE-EDTA (1:1 TrypLE-0.25mM mM EDTA/PBS) for $5 \mathrm{~min}$. HiPSCs were resuspended in $100 \mu \mathrm{L}$ of $1 \%$ BSA-PBS and incubated with the corresponding antibody for $30 \mathrm{~min}$ at $4{ }^{\circ} \mathrm{C}$ in the dark: BV510-conjugated Mouse Anti-human CD24 at 1:100 dilution (BD Horizon/ BD Biosciences \#563035), BV421-conjugated Mouse Anti-human CD57 at 1:100 dilution (BD Horizon/ BD Biosciences \#563896), PE-conjugated anti-human CD90 at 1:100 dilution (BD Biosciences \#555596), APC-conjugated Mouse Anti-human SSEA4 at 1:100 dilution (R\&D Systems \#FAB1435A), BV510-conjugated Mouse Anti-human CD77 at 1:100 dilution (BD Horizon/ BD Biosciences \#563630), APC-conjugated Mouse 
Anti-human CD130 at 1:40 dilution (BioLegend \#362006). Isotype-matched IgGs were used as negative controls. Thereafter, samples were washed twice with PBS and finally resuspended in FACS buffer (PBS 1mM EDTA, $25 \mathrm{mM}$ HEPES, 1\% BSA, pH7), to be recorded in a BD FACSCanto ${ }^{\mathrm{TM}}$ II with BD FACSDiva software. Flow cytometry data were analyzed using FlowJo software.

\section{Lentiviral production and cell transduction}

For lentiviral particle production, HEK293 cells were plated $5 \times 10^{6}$ cells $/ 10 \mathrm{~cm}$ dish (Corning \#430167) and the next day transfected with the FUtdTW TdTomato reporter plasmid (Addgene \#22478) together with two helper plasmids (psPax2 and PMD2G) using X-tremeGENE HP DNA Transfection Reagent (Roche). In brief, $400 \mu \mathrm{L}$ of OPTIMEM (Invitrogen) was mixed with $1 \mu \mathrm{g}$ PMD2G, $3 \mu \mathrm{g}$ psPax2 and $4 \mu \mathrm{g}$ lentiviral construction plasmid. $24 \mu \mathrm{L}$ of X-tremeGENE HP DNA Transfection Reagent was added and the mixture was incubated for 20 minutes at room temperature and gently applied to the cells. The next day, medium was replaced and lentiviral particle-containing supernatant was collected 36 hours later. Lentiviral particles were concentrated by ultracentrifugation $\left(2 \mathrm{~h}\right.$ at $20.000 \mathrm{~g}$ at $4^{\circ} \mathrm{C}$ ) and resuspended in PBS. Cells were transduced with the lentiviral particles, and 7 days later, tdTomato positive $\left(\right.$ tdTomato $^{+}$) cells were sorted using a BD FACSAria ${ }^{\mathrm{TM}} \mathrm{Ilu}$, analyzed with BD FACSdiva software.

\section{Mouse morula microinjection with 5iLAF hiPSCs}

Fertilized mouse embryos were harvested from superovulated B6.DBA2 female mice crossed with males of the same strain at 2.5 days postcoitum (dpc) by flushing the infundibulum with M2 medium (Sigma-Aldrich \#M7167-100ML). Human cells were dissociated with TrypLE-PBS-EDTA and magnetically selected to eliminate dead cells (Miltenyi Biotech \#130-090-101) and remove mouse feeders (Miltenyi Biotech \#130095-531) through LS Columns (Miltenyi Biotech \#130-042-401) following the manufacturer's instructions. Ten tdTomato ${ }^{+} 5$ iLAF hiPSCs were microinjected into the perivitelline space of the morula using Leica DMI3000 B microscope and mechanical micromanipulators with hanging joystick; TransferMan ${ }^{\circledR}$ NK2 (Eppendorf). After microinjection, embryos were cultured in $5 \mathrm{iLAF}$ medium for $48 \mathrm{~h}$ at $37^{\circ} \mathrm{C}$ under $5 \% \mathrm{O}_{2}$, $5 \% \mathrm{CO}_{2}$ in air (Drawer Type Incubator \#AD-3100), until they developed to the blastocyst stage. Then, tdTomato ${ }^{+}$hiPSCs inside morulas and blastocysts were photographed and quantified by eye under a fluorescence microscope. We statistically analyzed our sample using Kruskal-Wallis test with an adjusted p-value $<0.05$.

\section{Mouse embryos immunofluorescence staining}

Mouse embryos 0-, 24- and 48-h post-microinjection with human naïve 5iLAF cells were fixed with 4\% PFA solution in PBS for 45 min at room temperature, washed in PBS and permeabilized with PBS $0.1 \%$ TritonX-100 for $2 \mathrm{~h}$ at $4{ }^{\circ} \mathrm{C}$. Then, embryos were blocked in PBS 1\% BSA (Sigma-Aldrich \# A3912-100G) for $1 \mathrm{~h}$ at $4{ }^{\circ} \mathrm{C}$. Samples were stained with GATA3 (Abcam \#ab199428) at 1:50 in blocking solution overnight at $4^{\circ} \mathrm{C}$. After rinsing with PBS to remove the unbound antibodies, samples were exposed to the secondary antibody; Alexa Fluor ${ }^{\mathrm{TM}} 488$ goat anti-rabbit IgG (Invitrogen \#A11008) for 2 
hours at $4^{\circ} \mathrm{C}$ in darkness (dilution 1:500 in PBS). Nuclear counterstain was performed with Hoechst 33342 trihydrochloride (1:800) for $30 \mathrm{~min}$ at $4^{\circ} \mathrm{C}$ in darkness. Images were recorded with Confocal Scanning Laser Microscope (Zeiss LSM 800) and ZEN 2.3 system software. The contribution of 5iLAF hiPSCs to the trophectoderm linage was determined by co-expression of tdTomato and GATA3 within mouse embryos' orthogonal sections. Composite images were obtained with ZEN 2.3 system software.

\section{Pyrosequencing}

Pyrosequencing assay was designed to analyze DNA methylation in five $\mathrm{CpG}$ dinucleotides of LINE-1 human retrotransposon in primed and naïve hiPSCs. Sodium bisulfite modification of $1 \mu \mathrm{g}$ of genomic DNA was carried out with the CpGenome ${ }^{\mathrm{TM}}$ DNA Modification Kit (Chemicon \#S7820) following the manufacturer's protocol. After bisulfite treatment of DNA, PCR was performed using PyroMark master mix (QIAGEN) with a denaturalization at $95^{\circ} \mathrm{C}$ for $15 \mathrm{~min}$ and for 45 cycles consisting of denaturation at $94^{\circ} \mathrm{C}$ for $1 \mathrm{~min}$, annealing at $55^{\circ} \mathrm{C}$ for $1 \mathrm{~min}$, and extension at $72^{\circ} \mathrm{C}$ for $1 \mathrm{~min}$, followed by a final $10 \mathrm{~min}$ extension. The PCR reactions for pyrosequencing were done with a biotinylated specific reverse primer (LINE-Forward: 5'TTTTGAGTTAGGTGTGGGATATA-3'and LINE-Reverse: 5'-BiotinAAAATCAAAAAATT CCCTTTC-3'). The resulting biotinylated PCR products were immobilized to streptavidin Sepharose High Performance beads (GE healthcare, Uppsala, Sweden) and processed to yield high quality ssDNA using the PyroMark Vacuum Prep Workstation (Biotage, Uppsala, Sweden), according to the manufacturer's instructions. The pyrosequencing reactions were performed using the sequencing primer (LINE-Seq: 5'-AGTTAGGTGTGGGATATAGT-3') in the PyromarkTM ID (QIAGEN). Sequence analysis was performed using the PyroQ-CpG analysis software (QIAGEN). Human male genomic DNA universally methylated for all genes (Intergen Company, Purchase, NY) was used as a positive control for methylated alleles. Water blanks were included with each assay.

\section{Single-cell RNA-sequencing (scRNA-seq)}

Twenty-four hours before single-cell dissociation, 5iLAF human cells were treated with rock inhibitor Y27632 (5 $\mu \mathrm{M}$ - Axon Medchem \#1683). Next day, human cells were dissociated using 1:1 TrypLE (Gibco \#12605-028) 1X PBS and 0.25 mM EDTA (Invitrogen \#AM9260G) for $5 \mathrm{~min}$ at $37^{\circ} \mathrm{C}$ and resuspended in FACS buffer (1X PBS, 1 $\mathrm{mM}$ EDTA, $25 \mathrm{mM}$ HEPES $\mathrm{pH}=7$ and $1 \% \mathrm{BSA}$ ). Then, tdTomato ${ }^{+}$human cells were FACS sorted with BD FACSAria ${ }^{\mathrm{TM}} \mathrm{IIu}$ through a nozzle of $100 \mu \mathrm{m}$ in $1 \mathrm{X}$ PBS $0.05 \%$ BSA, and the number of cells was quantified in a Neubauer chamber.

The transcriptome of tdTomato+ naïve hiPSCs cultured under 5iLAF conditions for 3 passages was examined using NEXTGEM Single Cell 3' Reagent Kits v3 (10x Genomics) according to the manufacturer's instructions. 15,000 cells were loaded at a concentration of $700-1000$ cells $/ \mu \mathrm{L}$ on a Chromium Controller instrument (10X Genomics) to generate single-cell gel bead-in-emulsions (GEMs). In this step, each cell was encapsulated with primers containing a fixed Illumina Read 1 sequence, a cell-identifying 16 bp 10x barcode, a 10 bp Unique Molecular Identifier (UMI) and a poly-dT sequence. Upon cell 
lysis, reverse transcription yielded full-length, barcoded cDNA. This cDNA was then released from the GEMs, PCR-amplified and purified with magnetic beads (SPRIselect, Beckman Coulter). Enzymatic Fragmentation and Size Selection was used to optimize cDNA size prior to library construction. Fragmented cDNA was then end-repaired, Atailed and ligated to Illumina adaptors. A final PCR-amplification with barcoded primers allowed sample indexing. Library quality control and quantification was performed using Qubit 3.0 Fluorometer (Life Technologies) and Agilent's 4200 TapeStation System (Agilent), respectively. Sequencing was performed in a NextSeq500 (Illumina) (Read1: 28 cycles; Read2: 49 cycles; i7 index: 8 cycles) at an average depth of 45,000 reads/cell. The sequenced library was demultiplexed and converted to FASTQ files using the mkfastq function from Cell Ranger v5.0.1 (10x Genomics). Reads were aligned to the reference human genome (GRCh38) downloaded from the 10x Genomics website (version 2020-A) using the Cell Ranger (v5.0.1) count function with default parameters. Genome annotation corresponded to Ensembl v98. The median number of unique molecular identifiers (UMIs) and genes detected per cell were 20,302 and 4,537 respectively.

\section{scRNA-Seq analysis}

The computational analysis of the resulting UMI count matrices was performed using the R package Seurat (v 4.0.5) (Stuart et al., 2019). Cells were subjected to a quality control step, keeping those cells expressing between 1,000 and 6,500 genes, that had between 2,000 and 48,000 UMIs and with less than $10 \%$ of UMIs assigned to mitochondrial genes. Those thresholds were chosen upon visual inspection of distributions. A preliminary clustering retrieved a fibroblast population that was also removed from further analysis. Using this filtering, we kept 3,652 cells, with a median of 4,851 genes per cell. Genes detected in less than 3 cells were removed from the analyses.

The dataset was subjected to normalization, identification of highly variable features and scaling using the SCTransform function (Hafemeister and Satija, 2019), regressing out for the $\%$ of mitochondrial UMIs. To characterize the cell populations present, we performed an unsupervised clustering analysis using the Louvain algorithm with a resolution of 0.5 in a shared nearest neighbors graph constructed with the first 20 principal components, as implemented in the FindClusters and FindNeighbors Seurat functions. Non-linear dimensional reduction for visualization was done using the RunUMAP function with the same principal components. Cluster 5 was further subdivided using the FindSubCluster function with a 0.3 resolution. Cluster markers were identified using the FindAllMarkers function in the log-normalized counts by using the Wilcoxon Rank Sum test, keeping as differentially expressed those that presented an average $\log 2$ fold-change greater than 0.25 and an adjusted p-value $<0.05$. To perform overrepresentation analyses the R package enrichR (Kuleshov et al., 2016) was used with several databases, including GO_Biological_Process_2021 and WikiPathways_Human_2021. Signatures scores were obtained using the AddModuleScores function from Seurat. Genes in each signature were converted to their corresponding symbol from Ensembl v98. Cell cycle analysis was performed using the CellCycleScoring function with default parameters. The plots were 
generated using the DimPlot, DotPlot, VlnPlot and FeaturePlot functions from Seurat as well as the ggplot2, ComplexHeatmap and pheatmap R libraries.

\section{Integrated analysis with Liu dataset}

The scRNASeq datasets from (Liu et al., 2020) (RSeT, naïve, primed and days 0-7 of reprogramming) and this study were combined. Genes expressed in less than 10 cells and with an average expression lower than 0.01 were discarded. UMI counts were normalized using the NormalizedData function in Seurat and principal component analysis was performed using 2,000 variable features. The Harmony algorithm (Korsunsky et al., 2019) was used to integrate the samples with default parameters as implemented in the RunHarmony function, using the library correspondence as the only batch information for correction. UMAP embeddings were obtained using the first 30 batch-corrected principal components.

\section{RNA velocity}

The Cell Ranger output was processed using velocyto v0.17.17 (la Manno et al., 2018) with the 'run10x' mode to obtain a loom file including a count matrix of spliced and unspliced read counts. We merged this matrix together with a loom file generated from the Seurat analysis as input for scVelo v0.2.4 (Bergen et al., n.d.) choosing there the top 2,000 variable genes that share a minimum of 30 counts for spliced and unspliced mRNA. We learned the transcriptional dynamics of splicing kinetics and estimated the RNA velocity with the 'recover_dynamics' and 'velocity' (in dynamical mode) functions. We embedded the resulting velocities on the UMAP space obtained with Seurat with the 'velocity_embedding_stream' function.

\section{Transposable element analysis}

We used scTE (He et al., 2021) to quantify the expression of transposable elements. First, we built an index with the repetitive elements from hg38 in bed format and the annotation used for Cell Ranger (Ensembl v98) with scTE_build. Then, we ran scTE with the index and the output BAM file from Cell Ranger, obtaining a counts matrix including both genes and repetitive elements. We processed the matrix with Seurat as above, keeping the same cells and assigning the same clusters to find differentially expressed transposable elements and proportions of different families of repetitive elements.

\section{REFERENCES}

Beddington, R.S.P., Robertson, E.J., 1989a. An assessment of the developmental potential of embryonic stem cells in the midgestation mouse embryo. Development (Cambridge, England) 105, 733-737. https://doi.org/10.1242/DEV.105.4.733

Beddington, R.S.P., Robertson, E.J., 1989b. An assessment of the developmental potential of embryonic stem cells in the midgestation mouse embryo. Development (Cambridge, England) 105, 733-737. https://doi.org/10.1242/DEV.105.4.733

Bergen, V., Lange, M., Peidli, S., Wolf, F.A., Theis, F.J., n.d. Generalizing RNA velocity to transient cell states through dynamical modeling. Nature Biotechnology. https://doi.org/10.1038/s41587-020-0591-3 
Castel, G., Meistermann, D., Bretin, B., Firmin, J., Blin, J., Loubersac, S., Bruneau, A., Chevolleau, S., Kilens, S., Chariau, C., Gaignerie, A., Francheteau, Q., Kagawa, H., Charpentier, E., Flippe, L., François--Campion, V., Haider, S., Dietrich, B., Knöfler, M., Arima, T., Bourdon, J., Rivron, N., Masson, D., Fournier, T., Okae, H., Fréour, T., David, L., 2020. Induction of Human Trophoblast Stem Cells from Somatic Cells and Pluripotent Stem Cells. Cell reports 33. https://doi.org/10.1016/J.CELREP.2020.108419

Cervantes, R.B., Stringer, J.R., Shao, C., Tischfield, J.A., Stambrook, P.J., 2002. Embryonic stem cells and somatic cells differ in mutation frequency and type. Proceedings of the National Academy of Sciences of the United States of America 99, 3586. https://doi.org/10.1073/PNAS.062527199

Chan, Y.S., Göke, J., Ng, J.H., Lu, X., Gonzales, K.A.U., Tan, C.P., Tng, W.Q., Hong, Z.Z., Lim, Y.S., Ng, H.H., 2013. Induction of a human pluripotent state with distinct regulatory circuitry that resembles preimplantation epiblast. Cell stem cell 13, 663-675. https://doi.org/10.1016/J.STEM.2013.11.015

Cinkornpumin, J.K., Kwon, S.Y., Guo, Y., Hossain, I., Sirois, J., Russett, C.S., Tseng, H.W., Okae, H., Arima, T., Duchaine, T.F., Liu, W., Pastor, W.A., 2020. Naive Human Embryonic Stem Cells Can Give Rise to Cells with a Trophoblast-like Transcriptome and Methylome. Stem cell reports 15, 198-213. https://doi.org/10.1016/J.STEMCR.2020.06.003

Dong, C., Beltcheva, M., Gontarz, P., Zhang, B., Popli, P., Fischer, L.A., Khan, S.A., Park, K.M., Yoon, E.J., Xing, X., Kommagani, R., Wang, T., Solnica-Krezel, L., Theunissen, T.W., 2020. Derivation of trophoblast stem cells from naïve human pluripotent stem cells. eLife 9. https://doi.org/10.7554/ELIFE.52504

Gafni, O., Weinberger, L., Mansour, A.A., Manor, Y.S., Chomsky, E., Ben-Yosef, D., Kalma, Y., Viukov, S., Maza, I., Zviran, A., Rais, Y., Shipony, Z., Mukamel, Z., Krupalnik, V., Zerbib, M., Geula, S., Caspi, I., Schneir, D., Shwartz, T., Gilad, S., Amann-Zalcenstein, D., Benjamin, S., Amit, I., Tanay, A., Massarwa, R., Novershtern, N., Hanna, J.H., 2013. Derivation of novel human ground state naive pluripotent stem cells. Nature 504, 282-286. https://doi.org/10.1038/NATURE12745

Göke, J., Lu, X., Chan, Y.S., Ng, H.H., Ly, L.H., Sachs, F., Szczerbinska, I., 2015. Dynamic transcription of distinct classes of endogenous retroviral elements marks specific populations of early human embryonic cells. Cell stem cell 16, 135-141. https://doi.org/10.1016/J.STEM.2015.01.005

Grow, E.J., Flynn, R.A., Chavez, S.L., Bayless, N.L., Wossidlo, M., Wesche, D.J., Martin, L., Ware, C.B., Blish, C.A., Chang, H.Y., Pera, R.A.R., Wysocka, J., 2015. Intrinsic retroviral reactivation in human preimplantation embryos and pluripotent cells. Nature 522, 221-246. https://doi.org/10.1038/NATURE14308

Grundy, E.E., Diab, N., Chiappinelli, K.B., 2021. Transposable element regulation and expression in cancer. The FEBS journal. https://doi.org/10.1111/FEBS.15722

Guo, G., Stirparo, G.G., Strawbridge, S.E., Spindlow, D., Yang, J., Clarke, J., Dattani, A., Yanagida, A., Li, M.A., Myers, S., Özel, B.N., Nichols, J., Smith, A., 2021 a. Human naive epiblast cells possess unrestricted lineage potential. Cell Stem Cell 28, 1040-1056.e6. https://doi.org/10.1016/J.STEM.2021.02.025

Guo, G., Stirparo, G.G., Strawbridge, S.E., Spindlow, D., Yang, J., Clarke, J., Dattani, A., Yanagida, A., Li, M.A., Myers, S., Özel, B.N., Nichols, J., Smith, A., 2021b. Human naive epiblast cells possess unrestricted lineage potential. Cell stem cell 28, 1040-1056.e6. https://doi.org/10.1016/J.STEM.2021.02.025 
Guo, G., von Meyenn, F., Rostovskaya, M., Clarke, J., Dietmann, S., Baker, D., Sahakyan, A., Myers, S., Bertone, P., Reik, W., Plath, K., Smith, A., 2017. Epigenetic resetting of human pluripotency. Development (Cambridge, England) 144, 2748-2763. https://doi.org/10.1242/DEV.146811

Guo, G., von Meyenn, F., Santos, F., Chen, Y., Reik, W., Bertone, P., Smith, A., Nichols, J., 2016. Naive Pluripotent Stem Cells Derived Directly from Isolated Cells of the Human Inner Cell Mass. Stem cell reports 6, 437-446. https://doi.org/10.1016/J.STEMCR.2016.02.005

Hafemeister, C., Satija, R., 2019. Normalization and variance stabilization of single-cell RNA-seq data using regularized negative binomial regression. Genome Biology 20, 1-15. https://doi.org/10.1186/S13059-019-1874-1/FIGURES/6

He, J., Babarinde, I.A., Sun, L., Xu, S., Chen, R., Shi, J., Wei, Y., Li, Y., Ma, G., Zhuang, Q., Hutchins, A.P., Chen, J., 2021. Identifying transposable element expression dynamics and heterogeneity during development at the single-cell level with a processing pipeline scTE. Nature Communications 2021 12:1 12, 1-14. https://doi.org/10.1038/s41467-021-21808-x

Hendrickson, P.G., Doráis, J.A., Grow, E.J., Whiddon, J.L., Lim, J.W., Wike, C.L., Weaver, B.D., Pflueger, C., Emery, B.R., Wilcox, A.L., Nix, D.A., Peterson, C.M., Tapscott, S.J., Carrell, D.T., Cairns, B.R., 2017. Conserved roles of mouse DUX and human DUX4 in activating cleavage-stage genes and MERVL/HERVL retrotransposons. Nature genetics 49, 925-934. https://doi.org/10.1038/NG.3844

Houghton, F.D., Humpherson, P.G., Hawkhead, J.A., Hall, C.J., Leese, H.J., 2003. Na+, $\mathrm{K}+$, ATPase activity in the human and bovine preimplantation embryo.

Developmental biology 263, 360-366. https://doi.org/10.1016/J.YDBIO.2003.07.014

Idelevich, A., Vilella, F., 2020. Mother and Embryo Cross-Communication. Genes 2020, Vol. 11, Page 376 11, 376. https://doi.org/10.3390/GENES11040376

Io, S., Kabata, M., Iemura, Y., Semi, K., Morone, N., Minagawa, A., Wang, B., Okamoto, I., Nakamura, T., Kojima, Y., Iwatani, C., Tsuchiya, H., Kaswandy, B., Kondoh, E., Kaneko, S., Woltjen, K., Saitou, M., Yamamoto, T., Mandai, M., Takashima, Y., 2021. Capturing human trophoblast development with naive pluripotent stem cells in vitro. Cell stem cell 28, 1023-1039.e13. https://doi.org/10.1016/J.STEM.2021.03.013

Jiang, C.L., Jin, S.G., Lee, D.H., Lan, Z.J., Xu, X., O’Connor, T.R., Szabó, P.E., Mann, J.R., Cooney, A.J., Pfeifer, G.P., 2002. MBD3L1 and MBD3L2, two new proteins homologous to the methyl-CpG-binding proteins MBD2 and MBD3: characterization of MBD3L1 as a testis-specific transcriptional repressor. Genomics 80, 621-629. https://doi.org/10.1006/GENO.2002.7001

Kaneko, K.J., 2016. Metabolism of Preimplantation Embryo Development: A Bystander or an Active Participant? Current Topics in Developmental Biology 120, 259-310. https://doi.org/10.1016/BS.CTDB.2016.04.010

Korsunsky, I., Millard, N., Fan, J., Slowikowski, K., Zhang, F., Wei, K., Baglaenko, Y., Brenner, M., Loh, P. ru, Raychaudhuri, S., 2019. Fast, sensitive and accurate integration of single-cell data with Harmony. Nature methods 16, 1289-1296. https://doi.org/10.1038/S41592-019-0619-0

Kuleshov, M. v., Jones, M.R., Rouillard, A.D., Fernandez, N.F., Duan, Q., Wang, Z., Koplev, S., Jenkins, S.L., Jagodnik, K.M., Lachmann, A., McDermott, M.G., Monteiro, C.D., Gundersen, G.W., Ma'ayan, A., 2016. Enrichr: a comprehensive gene set enrichment analysis web server 2016 update. Nucleic acids research 44, W90-W97. https://doi.org/10.1093/NAR/GKW377 
la Manno, G., Soldatov, R., Zeisel, A., Braun, E., Hochgerner, H., Petukhov, V., Lidschreiber, K., Kastriti, M.E., Lönnerberg, P., Furlan, A., Fan, J., Borm, L.E., Liu, Z., van Bruggen, D., Guo, J., He, X., Barker, R., Sundström, E., CasteloBranco, G., Cramer, P., Adameyko, I., Linnarsson, S., Kharchenko, P. v., 2018. RNA velocity of single cells. Nature 2018 560:7719 560, 494-498. https://doi.org/10.1038/s41586-018-0414-6

Linneberg-Agerholm, M., Wong, Y.F., Herrera, J.A.R., Monteiro, R.S., Anderson, K.G.V., Brickman, J.M., 2019. Naïve human pluripotent stem cells respond to Wnt, Nodal and LIF signalling to produce expandable naïve extra-embryonic endoderm. Development (Cambridge, England) 146. https://doi.org/10.1242/DEV.180620

Liu, L., Leng, L., Liu, C., Lu, C., Yuan, Y., Wu, L., Gong, F., Zhang, S., Wei, X., Wang, M., Zhao, L., Hu, L., Wang, J., Yang, H., Zhu, S., Chen, F., Lu, G., Shang, Z., Lin, G., 2019a. An integrated chromatin accessibility and transcriptome landscape of human pre-implantation embryos. Nature communications 10. https://doi.org/10.1038/S41467-018-08244-0

Liu, L., Leng, L., Liu, C., Lu, C., Yuan, Y., Wu, L., Gong, F., Zhang, S., Wei, X., Wang, M., Zhao, L., Hu, L., Wang, J., Yang, H., Zhu, S., Chen, F., Lu, G., Shang, Z., Lin, G., 2019b. An integrated chromatin accessibility and transcriptome landscape of human pre-implantation embryos. Nature communications 10. https://doi.org/10.1038/S41467-018-08244-0

Liu, X., Nefzger, C.M., Rossello, F.J., Chen, J., Knaupp, A.S., Firas, J., Ford, E., Pflueger, J., Paynter, J.M., Chy, H.S., O’Brien, C.M., Huang, C., Mishra, K., Hodgson-Garms, M., Jansz, N., Williams, S.M., Blewitt, M.E., Nilsson, S.K., Schittenhelm, R.B., Laslett, A.L., Lister, R., Polo, J.M., 2017. Comprehensive characterization of distinct states of human naive pluripotency generated by reprogramming. Nature methods 14, 1055-1062. https://doi.org/10.1038/NMETH.4436

Liu, X., Ouyang, J.F., Rossello, F.J., Tan, J.P., Davidson, K.C., Valdes, D.S., Schröder, J., Sun, Y.B.Y., Chen, J., Knaupp, A.S., Sun, G., Chy, H.S., Huang, Z., Pflueger, J., Firas, J., Tano, V., Buckberry, S., Paynter, J.M., Larcombe, M.R., Poppe, D., Choo, X.Y., O’Brien, C.M., Pastor, W.A., Chen, D., Leichter, A.L., Naeem, H., Tripathi, P., Das, P.P., Grubman, A., Powell, D.R., Laslett, A.L., David, L., Nilsson, S.K., Clark, A.T., Lister, R., Nefzger, C.M., Martelotto, L.G., Rackham, O.J.L., Polo, J.M., 2020. Reprogramming roadmap reveals route to human induced trophoblast stem cells. Nature 586, 101-107. https://doi.org/10.1038/S41586-0202734-6

lo Nigro, A., de Jaime-Soguero, A., Khoueiry, R., Cho, D.S., Ferlazzo, G.M., Perini, I., Abon Escalona, V., Aranguren, X.L., Chuva de Sousa Lopes, S.M., Koh, K.P., Conaldi, P.G., Hu, W.S., Zwijsen, A., Lluis, F., Verfaillie, C.M., 2017. PDGFR $\alpha+$ Cells in Embryonic Stem Cell Cultures Represent the In Vitro Equivalent of the Pre-implantation Primitive Endoderm Precursors. Stem cell reports 8, 318-333. https://doi.org/10.1016/J.STEMCR.2016.12.010

Macfarlan, T.S., Gifford, W.D., Driscoll, S., Lettieri, K., Rowe, H.M., Bonanomi, D., Firth, A., Singer, O., Trono, D., Pfaff, S.L., 2012. Embryonic stem cell potency fluctuates with endogenous retrovirus activity. Nature 487, 57-63. https://doi.org/10.1038/NATURE11244

Maeso, I., Dunwell, T.L., Wyatt, C.D.R., Marlétaz, F., Veto, B., Bernal, J.A., Quah, S., Irimia, M., Holland, P.W.H., 2016. Evolutionary origin and functional divergence 
of totipotent cell homeobox genes in eutherian mammals. BMC biology 14 . https://doi.org/10.1186/S12915-016-0267-0

Meistermann, D., Bruneau, A., Loubersac, S., Reignier, A., Firmin, J., FrançoisCampion, V., Kilens, S., Lelièvre, Y., Lammers, J., Feyeux, M., Hulin, P., Nedellec, S., Bretin, B., Castel, G., Allègre, N., Covin, S., Bihouée, A., Soumillon, M., Mikkelsen, T., Barrière, P., Chazaud, C., Chappell, J., Pasque, V., Bourdon, J., Fréour, T., David, L., 2021. Integrated pseudotime analysis of human preimplantation embryo single-cell transcriptomes reveals the dynamics of lineage specification. Cell stem cell 28, 1625-1640.e6. https://doi.org/10.1016/J.STEM.2021.04.027

Messmer, T., von Meyenn, F., Savino, A., Santos, F., Mohammed, H., Lun, A.T.L., Marioni, J.C., Reik, W., 2019. Transcriptional Heterogeneity in Naive and Primed Human Pluripotent Stem Cells at Single-Cell Resolution. Cell reports 26, 815824.e4. https://doi.org/10.1016/J.CELREP.2018.12.099

Niakan, K.K., Eggan, K., 2013. Analysis of human embryos from zygote to blastocyst reveals distinct gene expression patterns relative to the mouse. Developmental biology 375, 54-64. https://doi.org/10.1016/J.YDBIO.2012.12.008

Ock, S.A., Knott, J.G., Choi, I., 2020. Involvement of CDKN1A (p21) in cellular senescence in response to heat and irradiation stress during preimplantation development. Cell stress \& chaperones 25, 503-508. https://doi.org/10.1007/S12192-020-01090-4

Okae, H., Toh, H., Sato, T., Suyama, M., Sasaki, H., Arima, T., 2018. Derivation of Human Trophoblast Stem Cells. https://doi.org/10.1016/j.stem.2017.11.004

Pastor, W.A., Chen, D., Liu, W., Kim, R., Sahakyan, A., Lukianchikov, A., Plath, K., Jacobsen, S.E., Clark, A.T., 2016. Naïve human pluripotent cells feature a methylation landscape devoid of blastocyst or germline memory. Cell stem cell 18, 323. https://doi.org/10.1016/J.STEM.2016.01.019

Petropoulos, S., Edsgärd, D., Reinius, B., Deng, Q., Panula, S.P., Codeluppi, S., Plaza Reyes, A., Linnarsson, S., Sandberg, R., Lanner, F., 2016. Single-Cell RNA-Seq Reveals Lineage and X Chromosome Dynamics in Human Preimplantation Embryos. Cell 165, 1012-1026. https://doi.org/10.1016/J.CELL.2016.03.023

Posfai, E., Rovic, I., Jurisicova, A., 2019. The mammalian embryo's first agenda: making trophectoderm. The International journal of developmental biology 63, 157-170. https://doi.org/10.1387/IJDB.180404EP

Rodriguez-Terrones, D., Gaume, X., Ishiuchi, T., Weiss, A., Kopp, A., Kruse, K., Penning, A., Vaquerizas, J.M., Brino, L., Torres-Padilla, M.E., 2018. A molecular roadmap for the emergence of early-embryonic-like cells in culture. Nature genetics 50, 106-119. https://doi.org/10.1038/S41588-017-0016-5

Senft, A.D., Macfarlan, T.S., 2021. Transposable elements shape the evolution of mammalian development. Nature reviews. Genetics 22, 691-711. https://doi.org/10.1038/S41576-021-00385-1

Shahbazi, M.N., 2020. Mechanisms of human embryo development: from cell fate to tissue shape and back. Development (Cambridge, England) 147. https://doi.org/10.1242/DEV.190629

Singh, H., Aplin, J.D., 2015. Endometrial apical glycoproteomic analysis reveals roles for cadherin 6, desmoglein-2 and plexin b2 in epithelial integrity. Molecular human reproduction 21, 81-94. https://doi.org/10.1093/MOLEHR/GAU087

Stirparo, G.G., Boroviak, T., Guo, G., Nichols, J., Smith, A., Bertone, P., 2018. Integrated analysis of single-cell embryo data yields a unified transcriptome 
signature for the human pre-implantation epiblast. Development (Cambridge, England) 145. https://doi.org/10.1242/DEV.158501

Stuart, T., Butler, A., Hoffman, P., Hafemeister, C., Papalexi, E., Mauck, W.M., Hao, Y., Stoeckius, M., Smibert, P., Satija, R., 2019. Comprehensive Integration of Single-Cell Data. Cell 177, 1888-1902.e21. https://doi.org/10.1016/J.CELL.2019.05.031

Suda, Y., Suzuki, M., Ikawa, Y., Aizawa, S., 1987. Mouse embryonic stem cells exhibit indefinite proliferative potential. Journal of Cellular Physiology 133, 197-201. https://doi.org/10.1002/JCP.1041330127

Takashima, Y., Guo, G., Loos, R., Nichols, J., Ficz, G., Krueger, F., Oxley, D., Santos, F., Clarke, J., Mansfield, W., Reik, W., Bertone, P., Smith, A., 2014. Resetting transcription factor control circuitry toward ground-state pluripotency in human. Cell 158, 1254-1269. https://doi.org/10.1016/J.CELL.2014.08.029

Theunissen, T.W., Friedli, M., He, Y., Planet, E., O’Neil, R.C., Markoulaki, S., Pontis, J., Wang, H., Iouranova, A., Imbeault, M., Duc, J., Cohen, M.A., Wert, K.J., Castanon, R., Zhang, Z., Huang, Y., Nery, J.R., Drotar, J., Lungjangwa, T., Trono, D., Ecker, J.R., Jaenisch, R., 2016. Molecular Criteria for Defining the Naive Human Pluripotent State. Cell stem cell 19, 502-515. https://doi.org/10.1016/J.STEM.2016.06.011

Theunissen, T.W., Powell, B.E., Wang, H., Mitalipova, M., Faddah, D.A., Reddy, J., Fan, Z.P., Maetzel, D., Ganz, K., Shi, L., Lungjangwa, T., Imsoonthornruksa, S., Stelzer, Y., Rangarajan, S., D’Alessio, A., Zhang, J., Gao, Q., Dawlaty, M.M., Young, R.A., Gray, N.S., Jaenisch, R., 2014. Systematic Identification of Culture Conditions for Induction and Maintenance of Naive Human Pluripotency. Cell Stem Cell 15, 471-487. https://doi.org/10.1016/J.STEM.2014.07.002

Töhönen, V., Katayama, S., Vesterlund, L., Jouhilahti, E.M., Sheikhi, M., Madissoon, E., Filippini-Cattaneo, G., Jaconi, M., Johnsson, A., Bürglin, T.R., Linnarsson, S., Hovatta, O., Kere, J., 2015. Novel PRD-like homeodomain transcription factors and retrotransposon elements in early human development. Nature communications 6. https://doi.org/10.1038/NCOMMS9207

Wang, Y., Zhao, C., Hou, Z., Yang, Y., Bi, Y., Wang, H., Zhang, Y., Gao, S., 2018. Unique molecular events during reprogramming of human somatic cells to induced pluripotent stem cells (iPSCs) at naïve state. eLife 7. https://doi.org/10.7554/ELIFE.29518

Ware, C.B., Nelson, A.M., Mecham, B., Hesson, J., Zhou, W., Jonlin, E.C., JimenezCaliani, A.J., Deng, X., Cavanaugh, C., Cook, S., Tesar, P.J., Okada, J., Margaretha, L., Sperber, H., Choi, M., Blau, C.A., Treuting, P.M., Hawkins, R.D., Cirulli, V., Ruohola-Baker, H., 2014. Derivation of naive human embryonic stem cells. Proceedings of the National Academy of Sciences of the United States of America 111, 4484-4489. https://doi.org/10.1073/PNAS.1319738111

Xiang, L., Yin, Y., Zheng, Y., Ma, Y., Li, Y., Zhao, Z., Guo, J., Ai, Z., Niu, Y., Duan, K., He, J., Ren, S., Wu, D., Bai, Y., Shang, Z., Dai, X., Ji, W., Li, T., 2020. A developmental landscape of 3D-cultured human pre-gastrulation embryos. Nature 577, 537-542. https://doi.org/10.1038/S41586-019-1875-Y

Yan, L., Yang, M., Guo, H., Yang, L., Wu, J., Li, Rong, Liu, P., Lian, Y., Zheng, X., Yan, J., Huang, J., Li, M., Wu, X., Wen, L., Lao, K., Li, Ruiqiang, Qiao, J., Tang, F., 2013. Single-cell RNA-Seq profiling of human preimplantation embryos and embryonic stem cells. Nature structural \& molecular biology 20, 1131-1139. https://doi.org/10.1038/NSMB.2660 
Zapata-Linares, N., Rodriguez, S., Mazo, M., Abizanda, G., Andreu, E.J., Barajas, M., Prosper, F., Rodriguez-Madoz, J.R., 2016. Generation and characterization of human iPSC line generated from mesenchymal stem cells derived from adipose tissue. Stem Cell Research 16, 20-23. https://doi.org/10.1016/J.SCR.2015.12.002 


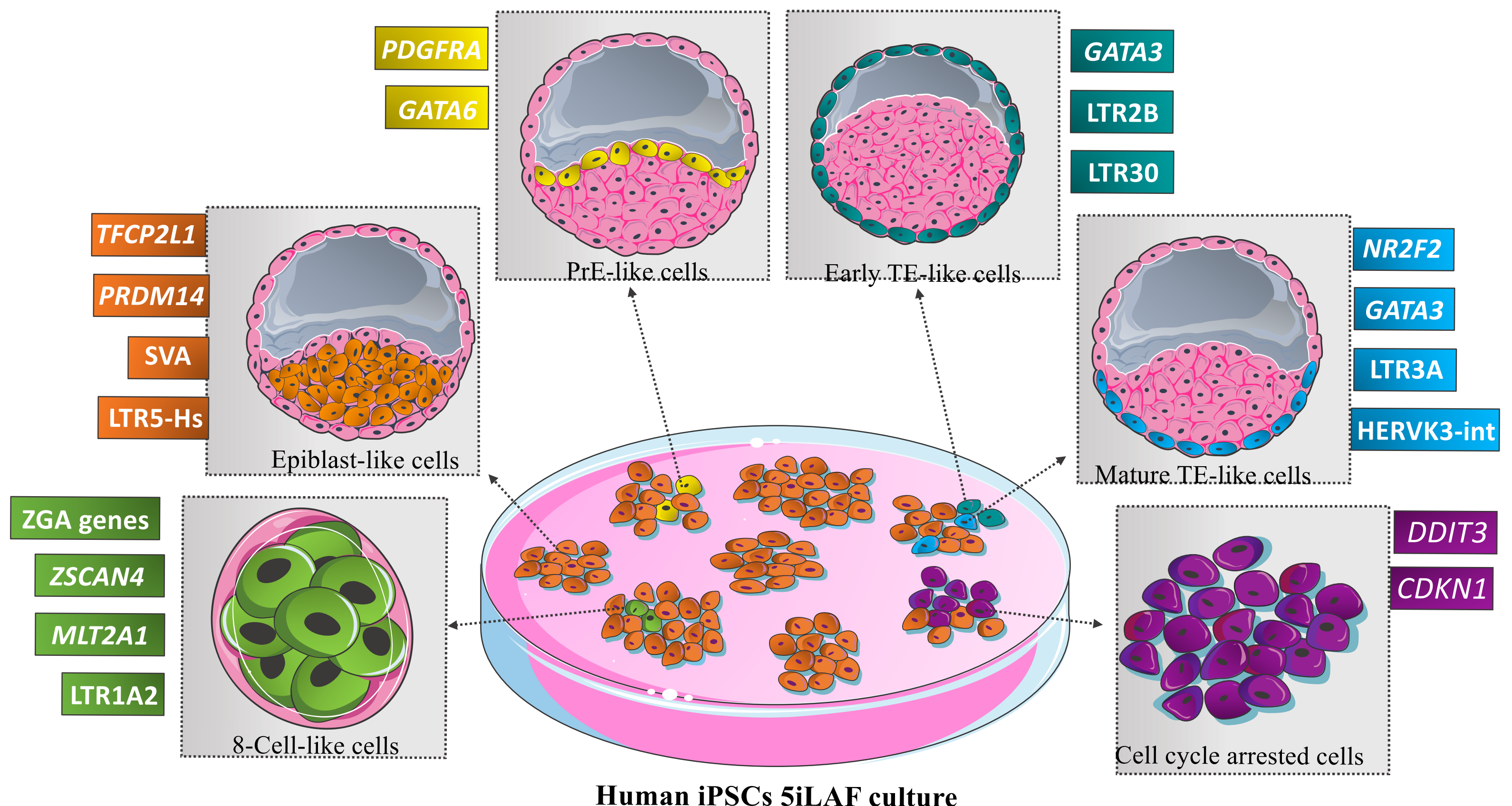


A

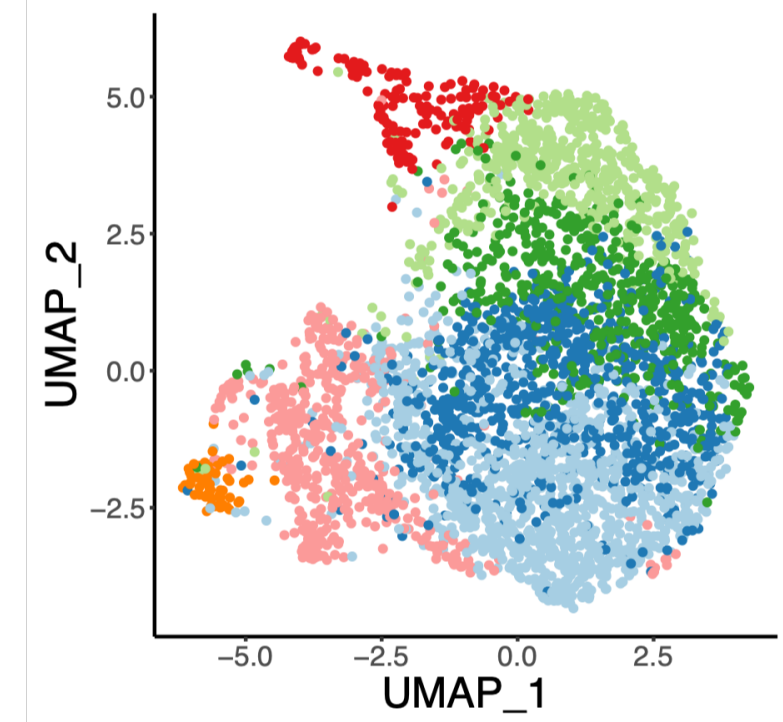

$001 \bigcirc 2 \bullet 3 \bigcirc 4 \bullet 5 \bigcirc 6$

D

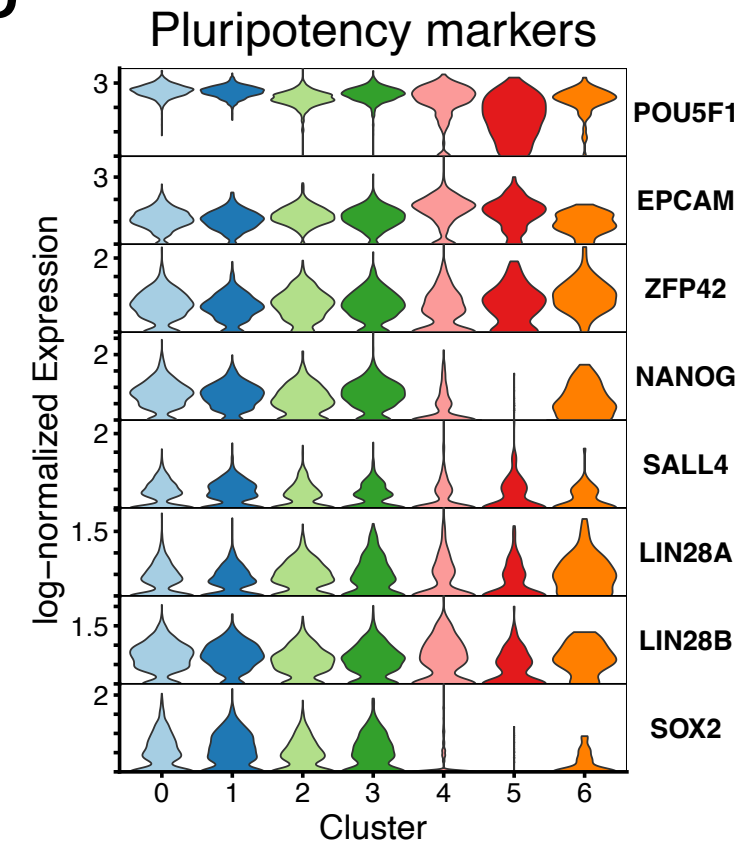

B

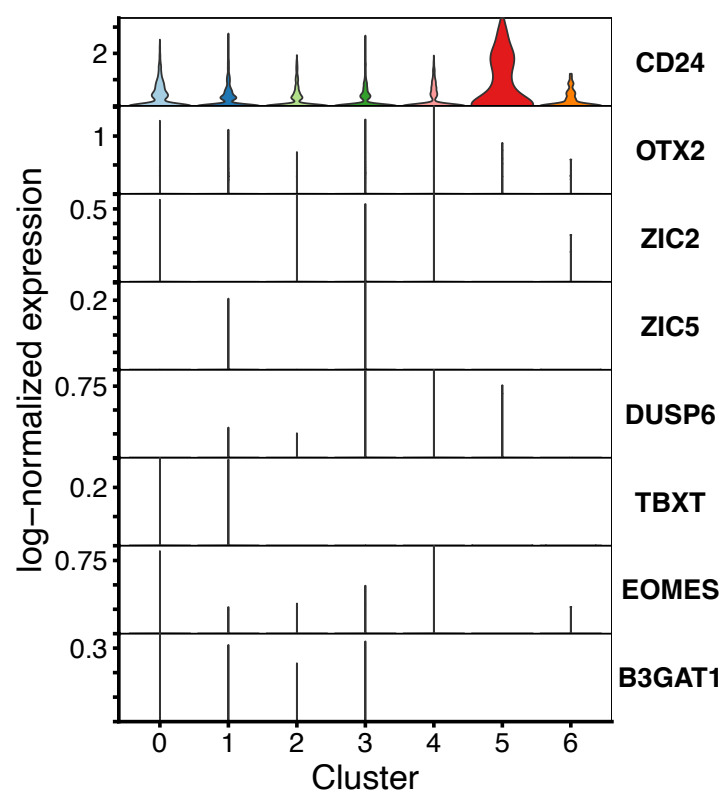

E

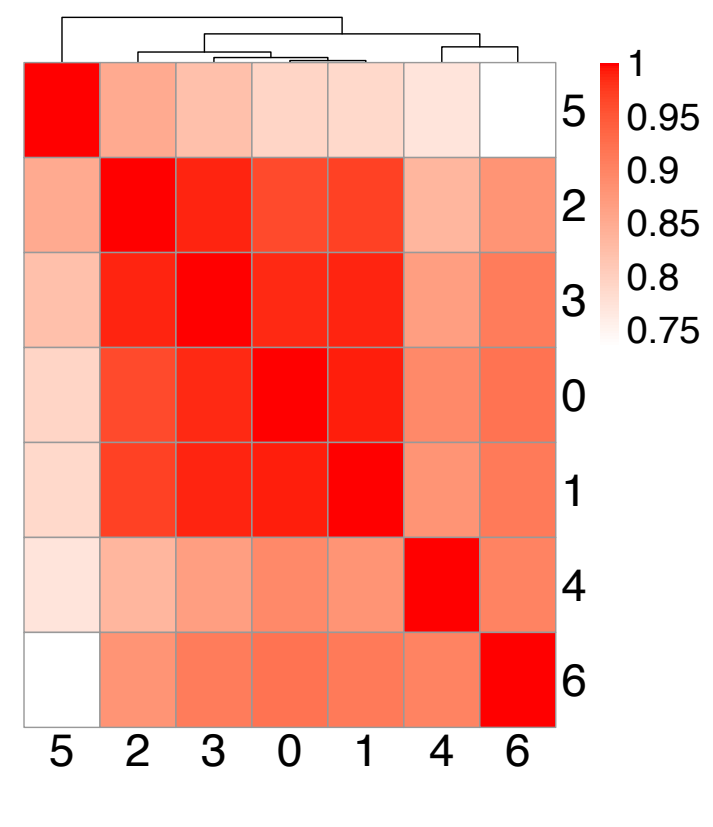

C

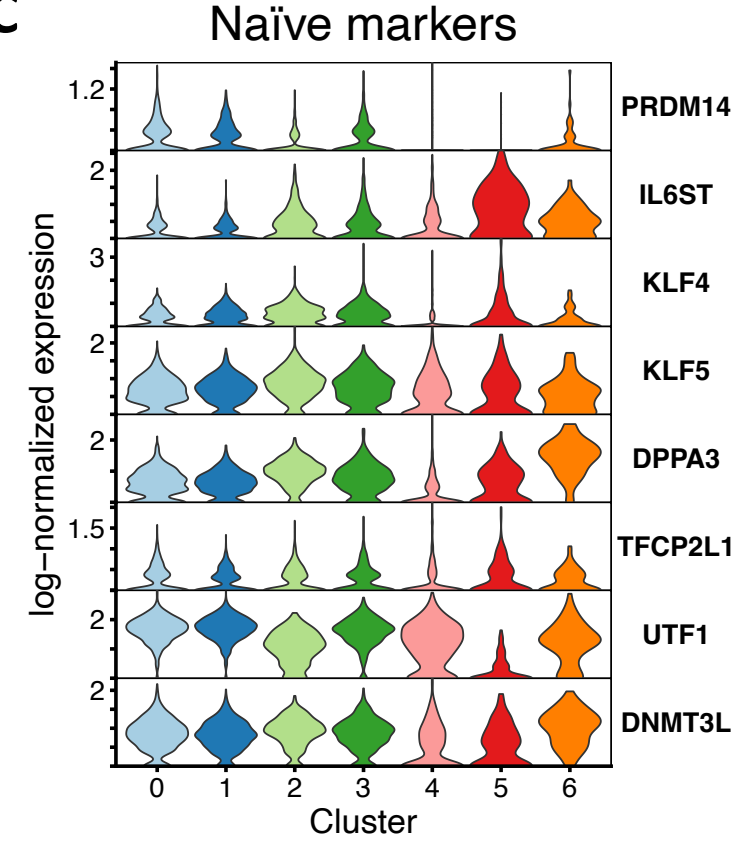

$\mathbf{F}$

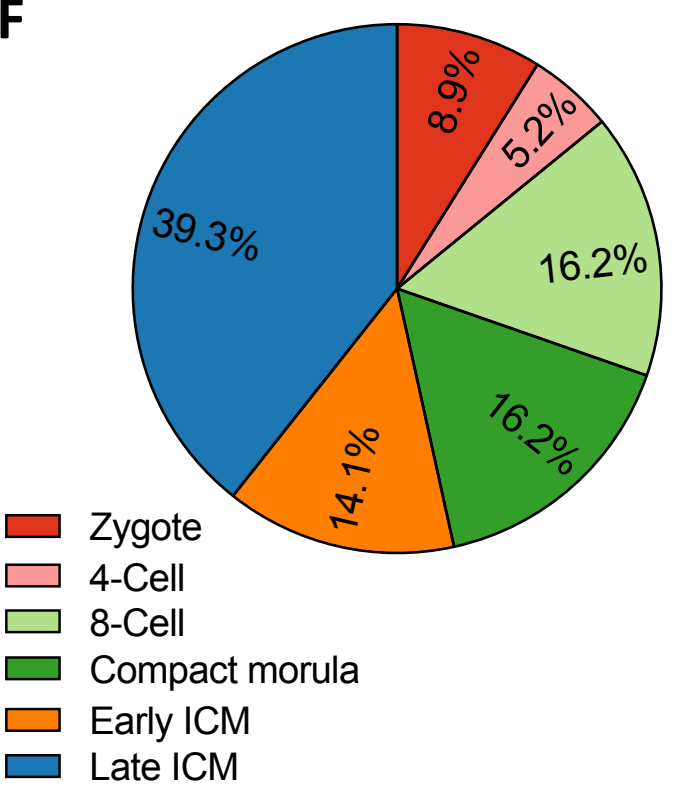

G
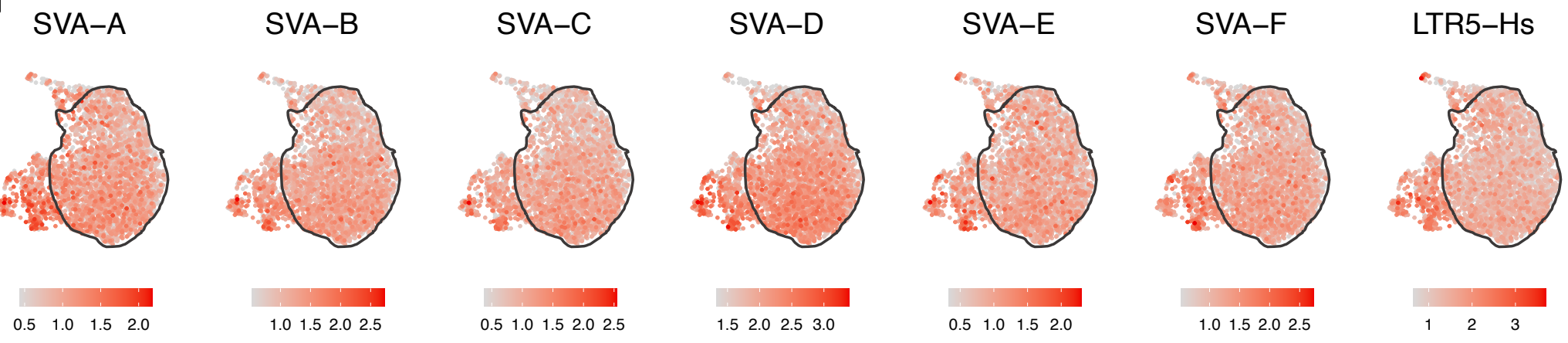

HERVK-int

Figure 1 
A
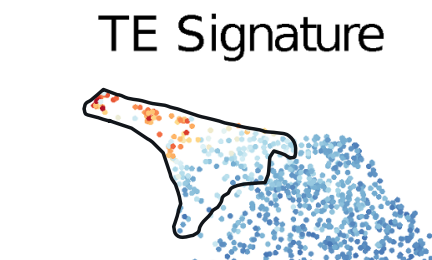

\%

$\therefore y^{2} y^{2}$
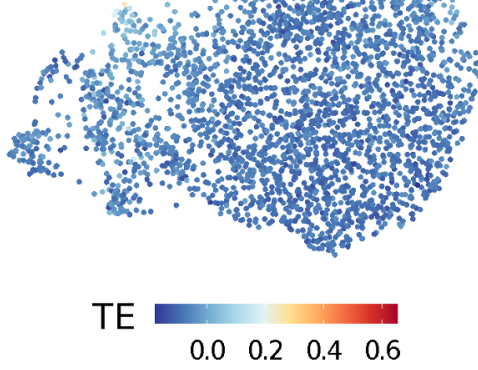

C

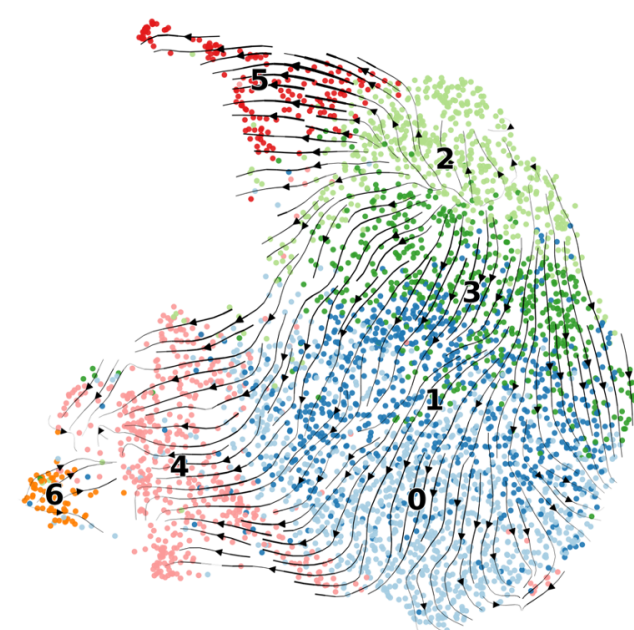

B

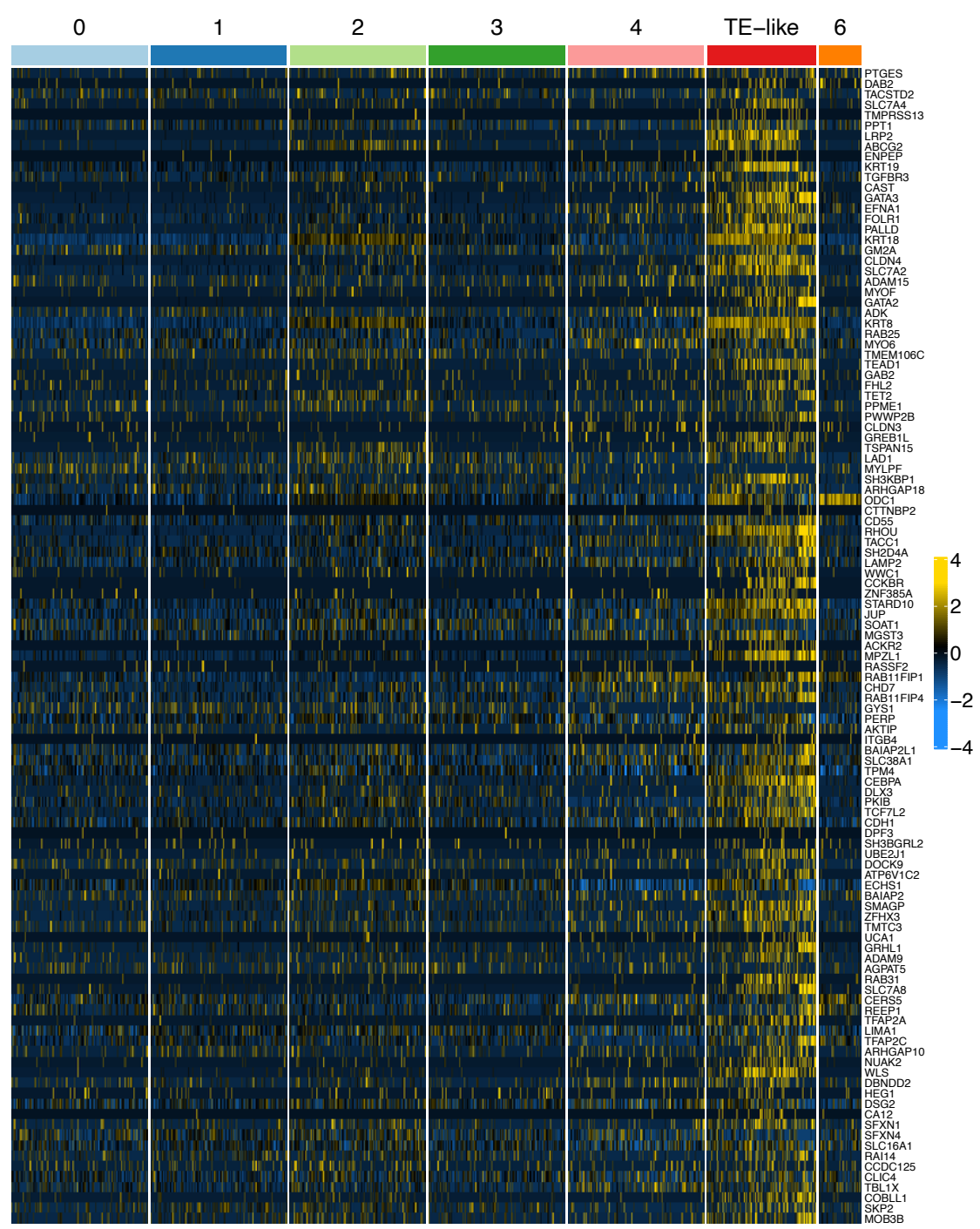

D

$\frac{4}{4}$

Hoechst

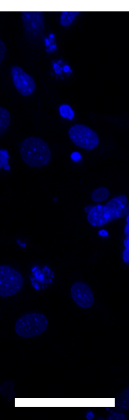

Hoechst

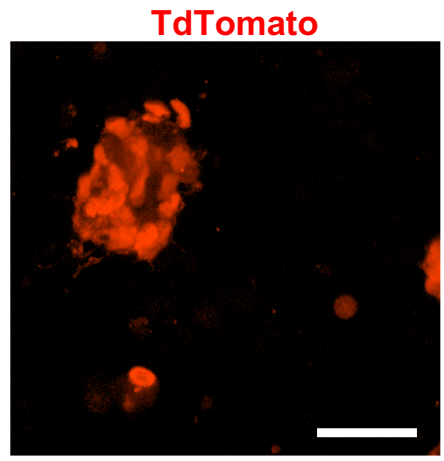

TdTomato

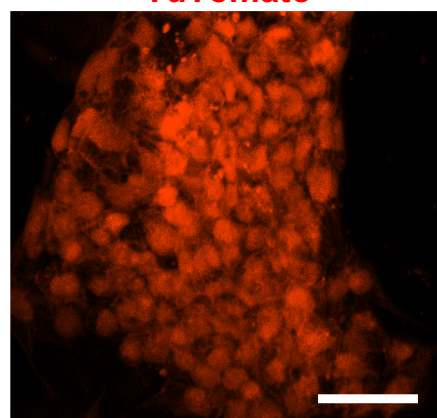

GATA3

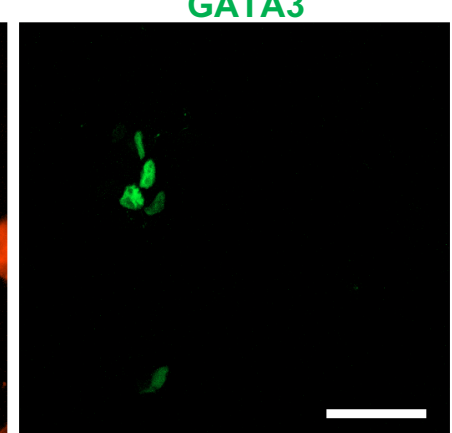

GATA3
Hoechst/TdTomato/GATA3

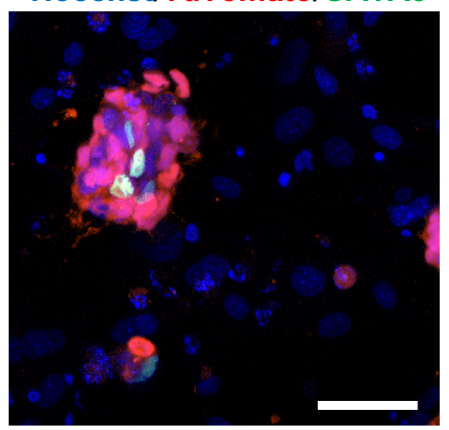

Hoechst/TdTomato/GATA3

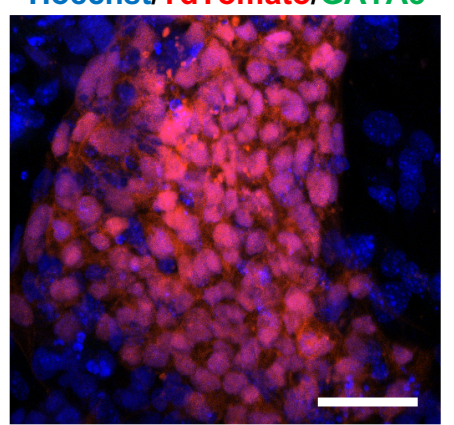

Figure 2 

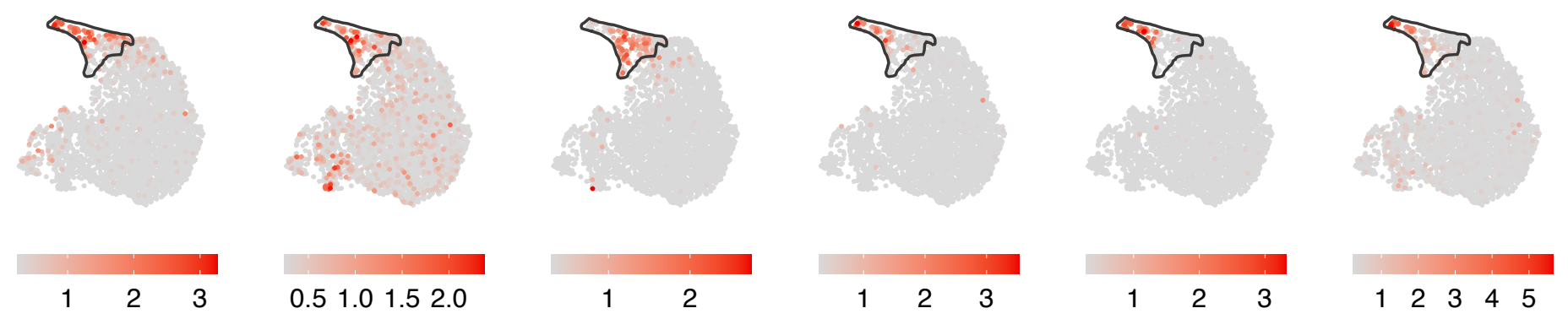

B
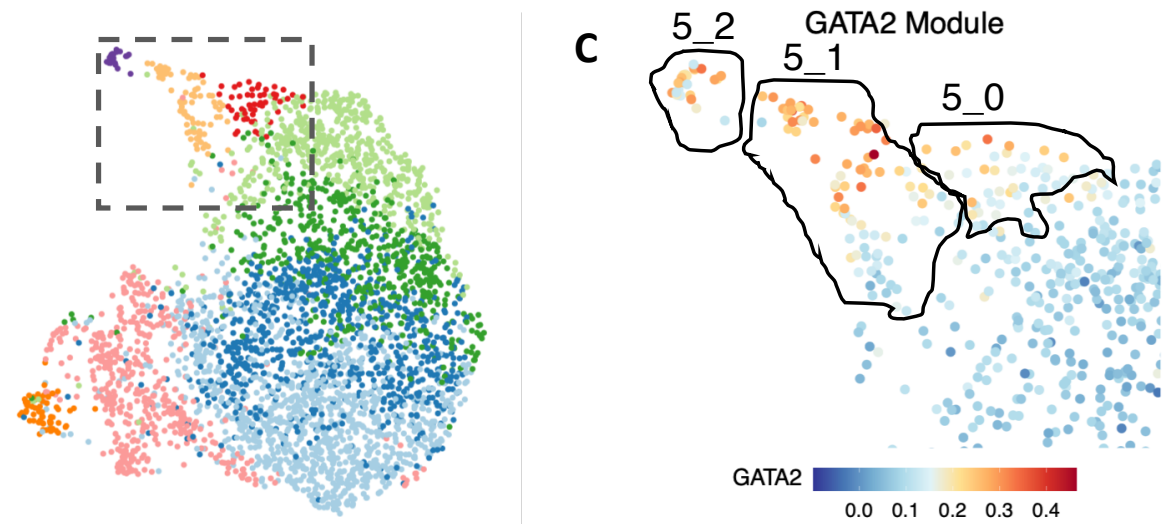

-5_0 Transition TE-like $\circ$ 5_1 Early TE-like -5_2 Mature TE-like

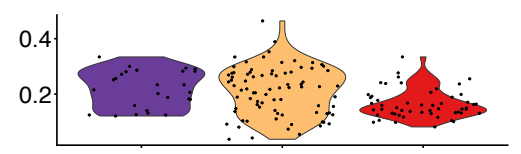

D
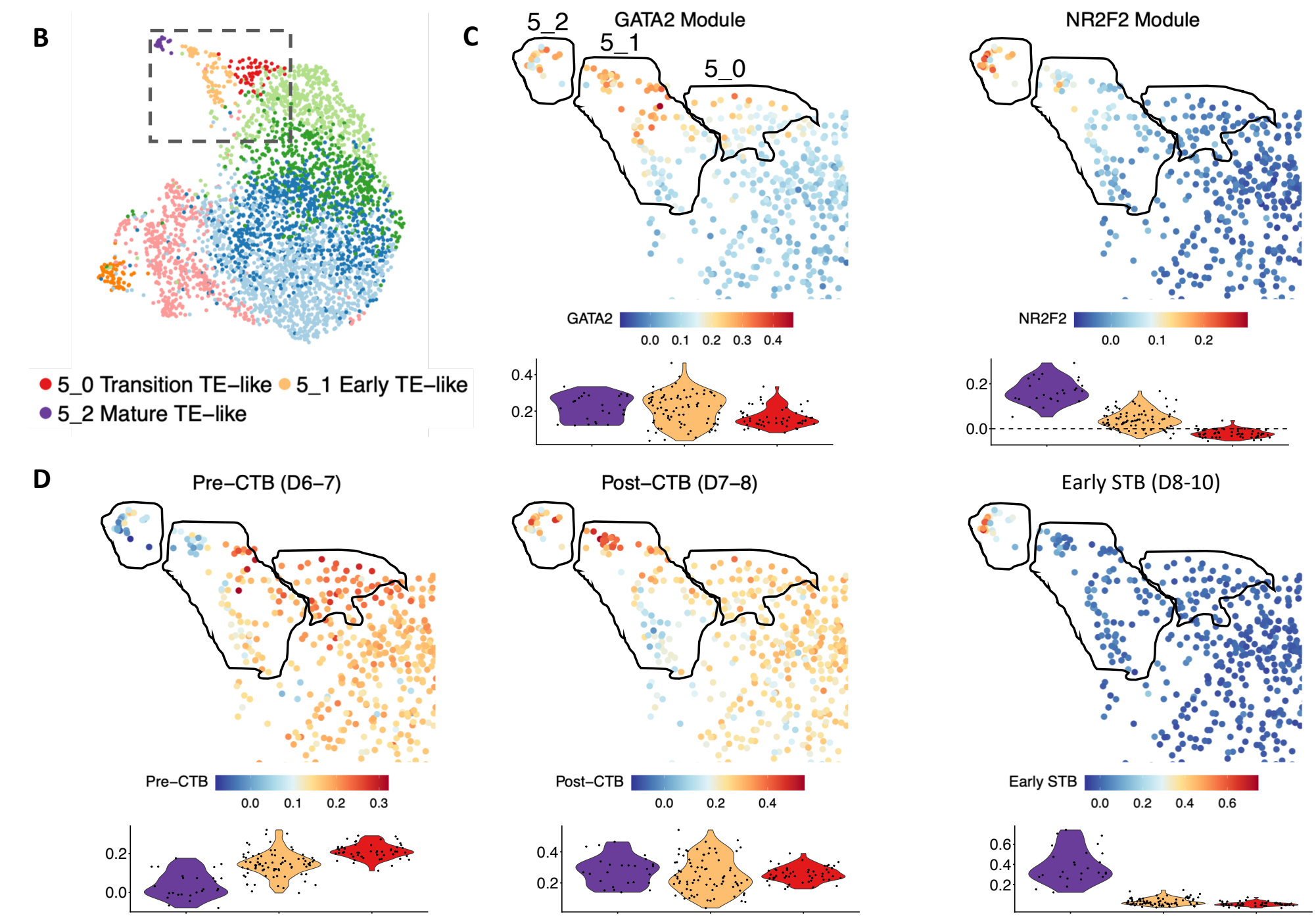

\section{E}
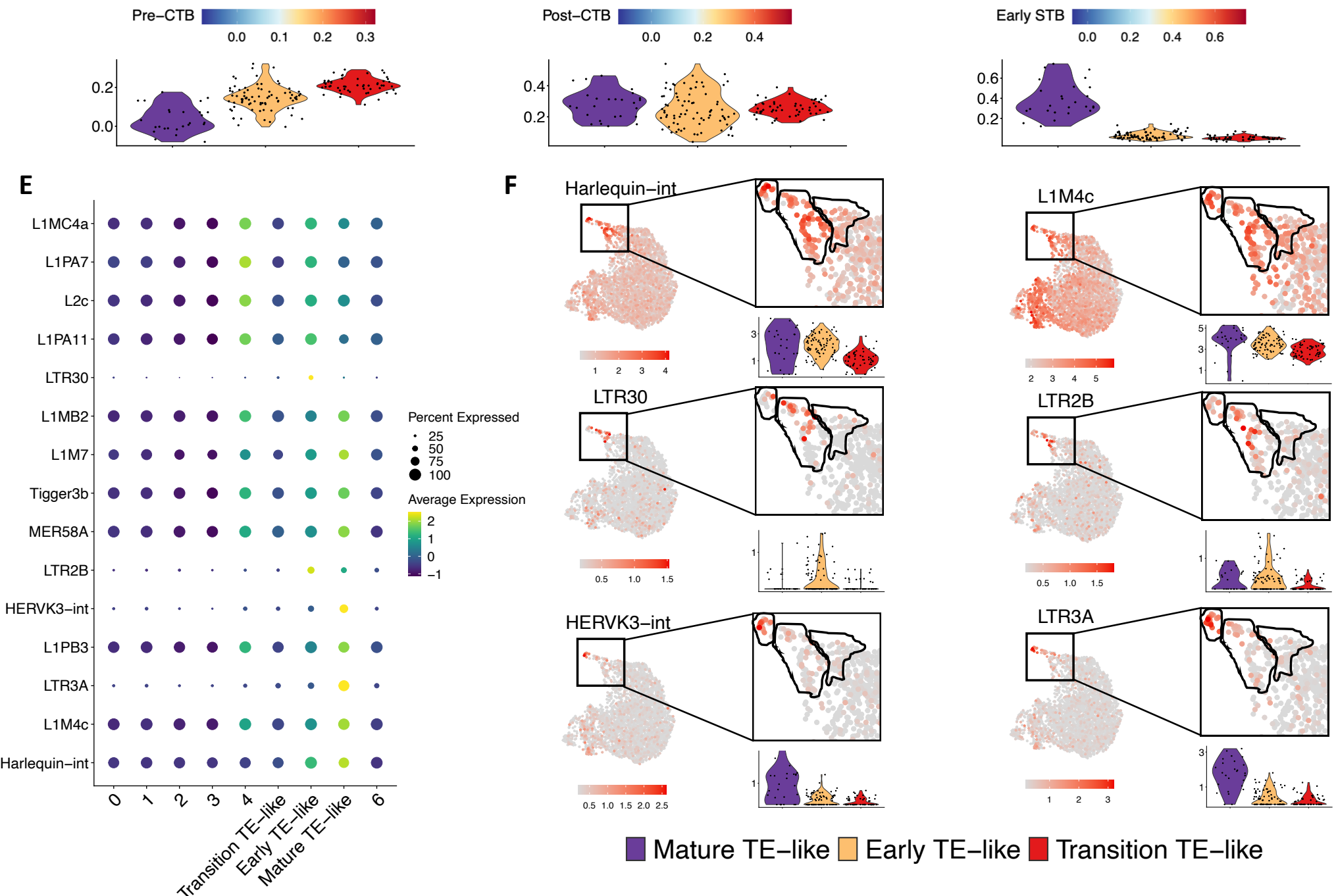

$\square$ Mature TE-like $\square$ Early TE-like $\square$ Transition TE-like

Figure 3 
A

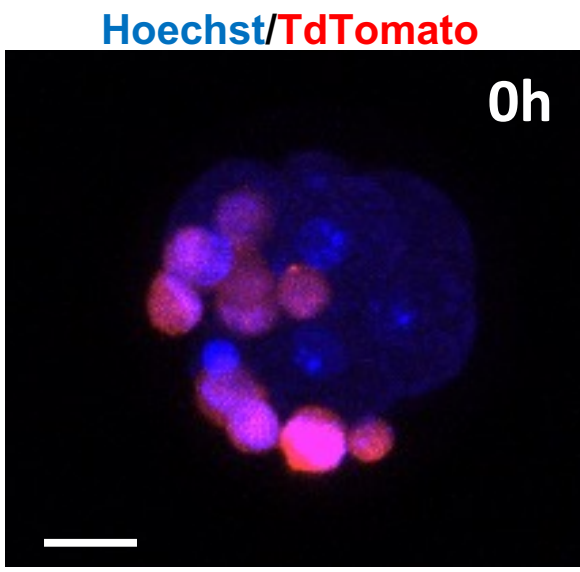

D

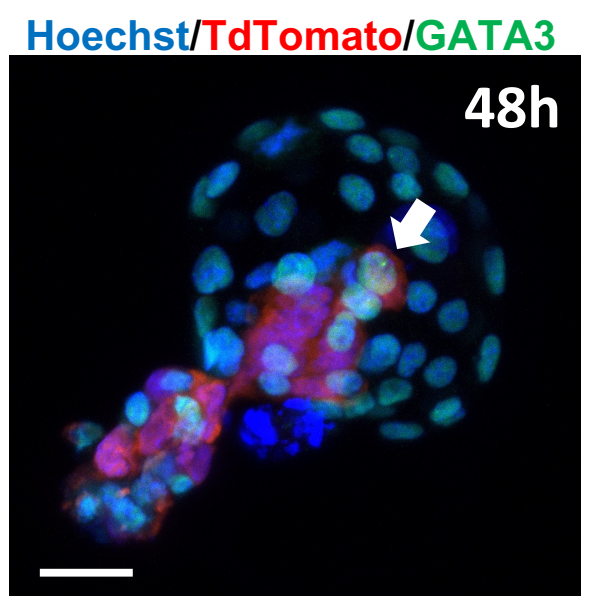

Hoechst/TdTomato

$48 h$

E

Hoechst/TdTomato/GATA3

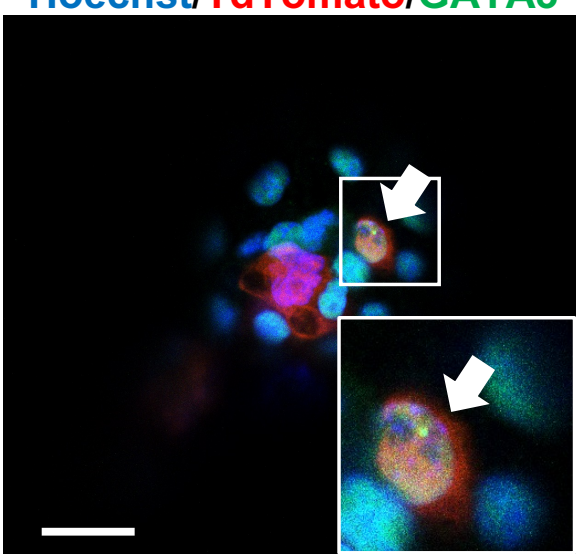

C

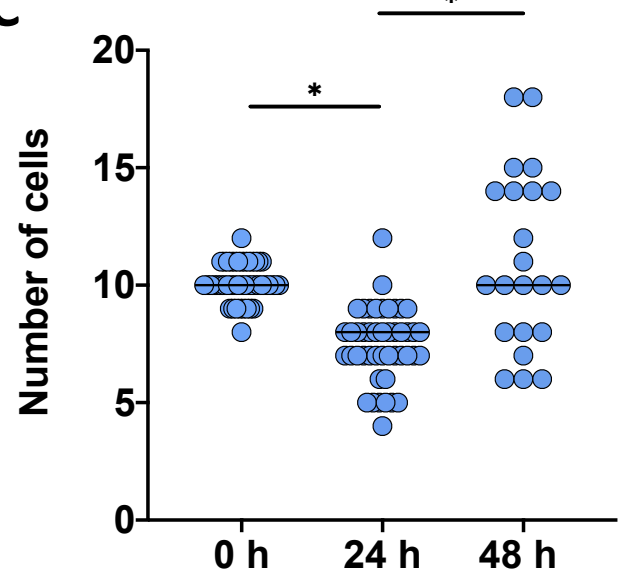

Time post-microinjection

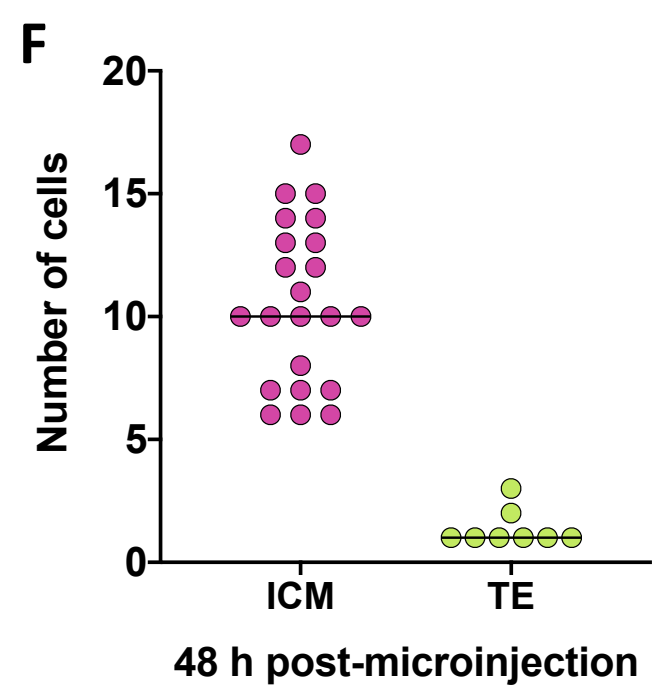

